\title{
A targeted next generation sequencing approach to develop robust, genotype-specific mutation profiles and uncover novel variants in Saccharomyces cerevisiae
}

Natalie A. Lamb ${ }^{1}$, Jonathan Bard ${ }^{2 ., 3}$, Michael J. Buck ${ }^{1,3}$, Jennifer A. Surtees ${ }^{1,3^{*}}$

${ }^{1}$ Department of Biochemistry, Jacobs School of Medicine and Biomedical Sciences, University at Buffalo (SUNY), Buffalo, NY, 14203

${ }^{2}$ University at Buffalo Genomics and Bioinformatics Core, Buffalo, NY, 14203

${ }^{3}$ Genetics, Genomics and Bioinformatics Graduate Program, Jacobs School of Medicine and Biomedical Sciences, University at Buffalo (SUNY), Buffalo, NY, 14203

* Corresponding author:

Jennifer A. Surtees

Department of Biochemistry

Jacobs School of Medicine and Biomedical Sciences

University at Buffalo, State University of New York

Rm 4215 - 955 Main Street, Buffalo, NY, 14203

Phone: (716) 829-6083; Fax, (716) 829-2725; Email: jsurtees@buffalo.edu 


\begin{abstract}
Distinct mutation signatures arise from environmental exposures and/or from defects in metabolic pathways that promote genome stability. The presence of a particular mutation signature in a cell or a tumor can therefore predict the underlying mechanism of mutagenesis, which, in practice, may be clinically important. These insults to the genome often alter dNTP pools, which itself impacts replication fidelity. Therefore, the impact of altered dNTP pools should be considered when making mechanistic predictions based on mutation signatures. We developed a targeted deep-sequencing approach on the CAN1 gene in Saccharomyces cerevisiae to define information-rich mutational profiles associated with distinct $r n r l$ backgrounds that alter replication fidelity by elevating dNTP levels.. The mutation spectra of $r n r l Y 285 F$ and rnr1Y285A alleles were characterized previously; our analysis was consistent with this prior work but the sequencing depth achieved in our study allowed a significantly more robust and nuanced computational analysis of the variants observed, generating profiles that integrated information about mutation spectra, position effects, and sequence context. This approach revealed novel, genotype-specific mutation profiles in the presence of even modest changes in dNTP pools. Furthermore, we identified broader sequence contexts and specific nucleotide motifs that influenced variant profiles in different $r n r l$ backgrounds, which allowed us to make specific mechanistic predictions about the impact of altered dNTP pools on replication fidelity.
\end{abstract}




\section{INTRODUCTION}

Specific environmental exposures and/or genetic backgrounds generate distinct mutation signatures (CHAN et al. 2012; CHAN et al. 2015; SAINI et al. 2020). Therefore, characterizing mutations provides insight into the underlying molecular mechanisms of mutagenesis in the absence of information about genotype or exposure. For example, $\mathrm{C} \rightarrow \mathrm{T}$ mutations have long been associated with UV exposure (HOWARD AND TESSMAN 1964). This is the basis for the Catalog of Somatic Mutations in Cancer (COSMIC), which has curated somatic mutation signatures in cancers to infer mutagenic mechanisms potentially active in generating the cancer (TATE et al. 2018). Our goal in this study was to develop a broader analytic pipeline that allowed us to build genotype-specific mutation profiles from the ground up, using both canonical and signature mutations (BRASH 2015) as well as sequence context.

In Saccharomyces cerevisiae, mutation spectra are frequently determined via one of two general strategies. The first requires selection of mutants, most often at the CAN1 locus (XU et al. 2008; KUMAR et al. 2011; BUCKLAND et al. 2014), which encodes an arginine permease that also imports the toxic arginine analog, L-canavanine, leading to cell death (FANTES AND CREANOR 1984; HoFFMANN 1985). Inactivating mutations in CAN1 block canavanine uptake and toxicity, allowing selection for mutations in CAN1. The can1 gene from individual resistant colonies is then amplified by PCR and subjected to Sanger sequencing. However, this approach is relatively low throughput (CHABES et al. 2003; XU et al. 2008; KUMAR et al. 2011) and will miss low frequency variants that might exist within a resistant colony. The second approach has been mutation accumulation (MA) experiments followed by WGS, which avoids potential bias associated with focusing on a single genetic locus (i.e. CAN1). However, the depth of sequencing is limited because the entire genome is sequenced, complicating statistically 
significant comparisons among genotypes (LUJAN et al. 2014; ZHU et al. 2014; RENTOFT et al. 2016). Increased depth requires increased numbers of MA lines and many passages are required to accumulate sufficient mutations and, again, low frequency and potentially diagnostic mutations will be missed.

We developed a high-throughput sequencing/bioinformatic pipeline that allowed characterization and comparison of robust mutation profiles from different genetic backgrounds. We focused on three $r n r 1$ alleles ( $r n r 1 D 57 N$, rnr1Y285F, rnr1Y285A) that decrease replication fidelity by altering dNTP pools (CHABES et al. 2003; XU et al. 2008; KUMAR et al. 2010). We chose these $r n r 1$ alleles because 1) altered ribonucleotide expression levels have been correlated with many cancers (AYE et al. 2015), 2) skewed dNTP pools have been observed in some cancer cell lines (MATHEWS 2015) and 3) there was Sanger and MA sequencing data available for comparison and validation of our analytic approach(XU et al. 2008; KUMAR et al. 2011; BUCKLAND et al. 2014; WATT et al. 2016). We paired selection for mutations at CAN1 with next-generation sequencing (NGS) to define mutation profiles for $R N R 1, \operatorname{rnr} 1 D 57 N, r n r 1 Y 285 F$ and $r n r 1 Y 285 A$. Importantly, $C A N 1$ has been found to be representative of mutations occurring genome-wide when Sanger and WGS approaches were compared (KUMAR et al. 2011;

BUCKLAND et al. 2014; WATT et al. 2016). This approach significantly increased sequencing depth compared with previous studies and allowed us to innovate in our analysis.

$R N R 1$ encodes the large subunit of ribonucleotide reductase (RNR), the enzyme that catalyzes the rate-limiting step in dNTP synthesis. RNR expression and activity is tightly regulated to promote replication fidelity and is misregulated in many different types of tumors and cancer cells (AYE et al. 2015; MATHEWS 2015; MATHEWS 2018). Altered RNR activity is associated with cancer and nucleobase analogs are frequently used as chemotherapeutics, 
commonly targeting RNR (JORDHEIM et al. 2013; KOHNKEN et al. 2015). Overexpression of Rrm2, encoding the small subunit of RNR, induced lung neoplasms in a mouse model, a result of an elevated mutator phenotype (XU et al. 2008). Similarly, mutations in SAMHD1, which encodes a dNTP triphosphohydrolase that modulates dNTP pools in mammalian systems, led to oncogenesis likely driven by increases in dNTP pool levels (RENTOFT et al. 2016; MAUNEY AND HoLLIS 2018). Mutations that affect the allosteric regulation of RNR ( $n n r 1 D 57 N, r n r 1 Y 285 F$, rnr1Y285A) increase and/or skew dNTP pools and increase mutation rates to varying degrees (CHABES et al. 2003; XU et al. 2008; KUMAR et al. 2010; KUMAR et al. 2011; BUCKLAND et al. 2014; WATT et al. 2016). Altered dNTP pools compromise replication fidelity by: 1) increasing the frequency of misinsertion and misalignment by replicative DNA polymerases and 2) biasing those polymerases toward synthesis at the expense of proofreading, promoting extension beyond a mispair (PHEAR et al. 1987; KUNKEL AND SONI 1988; KUMAR et al. 2010).

In this study we defined and characterized mutation profiles in wildtype yeast and in isogenic strains bearing the rnr1D57N, rnr1Y285F and rnr1Y285A alleles (CHABES et al. 2003; XU et al. 2008; KUMAR et al. 2010). We built comprehensive mutation profiles from first principles, which consisted of mutation spectrum analysis of the specific types of variants observed and their position, the trinucleotide context in which the variants occurred and the broader sequence context of the variants, identifying motifs that were specifically enriched or depleted in distinct $r n r l$ backgrounds. Importantly, and in contrast to previous work (CHABES et al. 2003; XU et al. 2008; KUMAR et al. 2010; KUMAR et al. 2011; BUCKLAND et al. 2014; WATT et al. 2016), our analysis defined novel, distinct profiles in all three $r n r l$ backgrounds, with rnrlY285A exhibiting the most unique profile. We noted many of the same rnr1Y285A mutation motifs determined by WGS (WATT et al. 2016), while also revealing new high and low 
frequency variants for $r n r l Y 285 A$ and unique profiles in $r n r 1 Y 285 F$ and $r n r 1 D 57 N$, indicating that even small changes in dNTP pools contribute to mutagenesis.

\section{MATERIALS AND METHODS}

\section{Strains and plasmids}

All strains in this study were derived from the W303 RAD5+ background (Table S1). Strains containing $r n r 1 Y 28 F / A$ in the $p G A L-R N R I$ background and integration plasmids to re-create these strains (KUMAR et al. 2010) were kindly provided by Andrei Chabes. To integrate rnrl alleles at the endogenous $R N R 1$ locus in the absence of $p G A L-R N R 1$, we created a new set of rnrl integration plasmids. First, we amplified pRS416 (SIKORSKI AND HIETER 1989) with SO261 and SO262, which each contain an AatII recognition site (Table S2). The resulting PCR product, which consists of pRS416 without the ARS/CEN region, was digested with AatII and ligated to generate the yeast integration plasmid, pEM3, which carries a counter-selectable URA3 marker (Table S3). RNR1 was amplified from JSY13 with SO263 (Table S2; encodes a XhoI site) and SO265 (Table S2; encodes a SpeI site) to amplify a product that extends $\sim 1 \mathrm{~kb}$ upstream of the endogenous $R N R 1$ start site to $\sim 3 \mathrm{~kb}$ downstream of the $R N R 1$ start site, to capture the full RNRI gene. This fragment was digested with XhoI and SpeI and ligated into pEM3 to generate the RNR1 integration plasmid pNL1 (Table S3). To generate a truncated version of $R N R I$ in the same plasmid, $R N R I$ was amplified from JSY13 with SO263 (encodes a XhoI site) and SO264 (Table S2; encodes a SpeI site) to amplify a product that extends $\sim 1 \mathrm{~kb}$ upstream of the endogenous $R N R I$ start site to $\sim 2 \mathrm{~kb}$ into the $R N R I$ open reading frame. The PCR product was digested with XhoI and SpeI and ligated into pEM3 to generate pNL2 (Table S3). 
We generated versions of pNL1 and pNL2 that encode $r n r 1 D 57 N$, rnrlY285F and rnr1Y285A using the Q5 site-directed mutagenesis kit (New England Biolabs) (Table S3). Oligonucleotides used for the mutagenesis are described in Table S2. Plasmids were sequenced to confirm the presence of the desired mutations and absence of secondary mutations. To integrate each $r n r l$ allele into the endogenous $R N R l$ locus, both the full-length and truncated integration plasmid for a given allele were linearized with Sph1 and used to co-transform JSY13 using the standard lithium acetate approach (GIETZ et al. 1992). Transformants were selected on synthetic complete medium lacking uracil (SC-URA). Single colonies were patched onto YPD to allow for homologous recombination and transplacement of one copy of $r n r 1$. After 2 days growth at $30^{\circ} \mathrm{C}$, cells from these patches were struck out to obtain single colonies on plates containing 5-FOA $(0.1 \%)$, selecting for a loss of $U R A 3$. Plasmid integration was confirmed by PCR using SO266 (Table S2; specific to the integration plasmid) and SO230 (Table S2; specific to the genomic locus). Sanger sequencing was used to confirm retention of a mutant $r n r 1$ allele and a loss of RNR1. rnrl was amplified in two fragments for sequencing, using the SO32/SO21 and SO25/SO22 primer pairs (Table S2).

\section{Mutation Rates at can1}

Mutation rates were determined through canavanine resistance assays as described previously (XU et al. 2008). Strains were struck out to single colonies on complete media. individual $2 \mathrm{~mm}$ colonies were carefully measured and selected to assay. Colonies were suspended in $100 \mu \mathrm{l}$ in TE (10 mM Tris-HCl, $\mathrm{pH} 7.5 ; 1 \mathrm{mM}$ EDTA); $75 \mu$ of undiluted colony suspension was plated on SC-ARG +canavanine plates. The cell suspension was diluted 1:10,000 and $20 \mu \mathrm{l}$ was plated under permissive conditions on SC-ARG. At least two independent isolates 
of 11 colonies were assayed for each genotype. Isolates were first analyzed independently before grouping data to calculate rates. Mutation rates and confidence intervals were calculated utilizing FluCalc fluctuation analysis software [Radchenko 2018].

\section{Pooling Canavanine resistant colonies}

Strains were patched on SC-ARG plates and grown at $30^{\circ} \mathrm{C}$ for 3 days, $\sim 30-35$ generations. A quarter of the patch was used to inoculate a $25 \mathrm{~mL} \mathrm{SC}$-ARG liquid culture. A patch was used to inoculate the culture instead of a single colony to reduce the potential effects of jackpot mutations in the cultures. Cells were grown for approximately 3-4 additional generations and then plated on SC-ARG + canavanine plates, to select for approximately 2,000 canavanine resistant $\left(\mathrm{Can}^{\mathrm{R}}\right)$ colonies. It required another $\sim 30$ generations to generate colonies, for a total of 65-70 generations of accumulated mutations, although mutations that confer canavanine resistance should occur in the first 35-40 generations. Canavanine resistant colonies were selected in at least four independent experiments per genotype. For each genotype, at least two independent isolates were used (Table S4).

Colonies were counted, and collected by adding TE (100 mM Tris-HCl, pH 7.4, $10 \mathrm{mM}$ EDTA) to the plate and using a sterile glass spreader to scrape off the cells. Colonies from multiple plates were pooled and resuspended in TE ( $\mathrm{pH} 7.4)$ to a final volume of $10-12 \mathrm{~mL}$. One $\mathrm{mL}$ of the colony suspension was used to extract genomic DNA (gDNA). Briefly, cells were lysed by vortexing in $200 \mu \mathrm{l}$ 1:1 phenol: chloroform, $200 \mu \mathrm{l}$ chromosome preparation buffer (10 mM Tris,-HCl, $\mathrm{pH}$ 8.0, $100 \mathrm{mM} \mathrm{NaCl}, 1 \mathrm{mM}$ EDTA, 1\% SDS, 2\% Triton X-100) and 0.3 grams acid-washed glass beads (Sigma; 425-600 microns). $200 \mu \mathrm{l}$ TE pH 8.0 was added and reactions were centrifuged for 5 minutes at $16,000 \mathrm{xg}$. The resulting supernatant was collected. Three 
additional phenol:chloroform extractions were performed to increase DNA purity. gDNA was

precipitated by addition of ammonium acetate to a final concentration of $100 \mathrm{mM}$ followed by 2 volumes of $95 \%$ ethanol. The gDNA was collected by centrifugation at $16,000 \mathrm{xg}$ for 10 minutes, followed by a wash with $70 \%$ ethanol. The gDNA pellets were resuspended in $50 \mu 1$ nuclease free water with RNase A (final concentration of $50 \mu \mathrm{g} / \mathrm{ml}$ ) and incubated at room temperature for at least 1 hour after which gDNA was stored at $-20^{\circ} \mathrm{C}$.

\section{Pooling unselected samples}

In addition to canavanine resistant colonies, we also pooled $\sim 2000$ unselected colonies from RNR1, rnrlD57N, rnrlY285F and rnrlY285A backgrounds as permissive controls. These colonies were grown as described above, except that in the final step, cells were grown on SCARG in the absence of canavanine. Colonies were pooled and genomic DNA extracted as described above.

\section{Library Preparation and Sequencing}

CAN1/canl was amplified from gDNA in 6 overlapping, 349-350 base pair fragments using primers listed in Table S2. KAPA HiFi ReadyMix (Roche) was used to amplify these fragments in $25 \mu \mathrm{L}$ reactions for each canl region for each of the 150 samples (total of 906 reactions). Two- $\mu \mathrm{L}$ of DNA was added to each reaction, in the range of 50-500 ng. Two- $\mu \mathrm{L}$ of each PCR reaction was electrophoresed on a $1 \%$ agarose gel to confirm amplification. For each sample, 20 $\mu \mathrm{L}$ of PCR product from each of the 6 regions were pooled in a 96-well plate and purified using the Zymo ZR-96 DNA Clean-up Kit. A total of 65 pooled sample sets (Table S4) were generated 
for paired-end sequencing $(2 \times 300)$, including technical replicates. PCR products from the same genomic preparation of pooled samples were independently amplified and sequenced.

\section{Library barcoding and QC}

Nextera barcode adapters were added to canl amplicons and were then minimally PCR amplified (8 cycles) for attachment of Illumina Nextera XT index primers set A (Illumina). After PCR, excess adapters were removed using Ampure XP beads (Beckman Coulter) and samples were eluted into EB buffer. Barcoded amplicons were checked for quality using an Advanced Analytical Fragment Analyzer and Qubit Fluorescence (Invitrogen). Amplicons were pooled to $10 \mathrm{nM}$ in $\mathrm{EB}$ buffer and the final concentration was determined using the Illumina Universal qPCR Amplification kit from Kapa Biosystems. All pooled samples were diluted to $4 \mathrm{nM}$, denatured using $\mathrm{NaOH}$ and loaded onto an Illumina MiSeq sequencing platform (PE300, V3) with $20 \%$ PhiX control. The sequencing was performed in two separate runs to increase coverage and as a check for reproducibility.

\section{Upstream sequencing analysis}

Reads were trimmed using a variable length trimmer (CutAdapt version 1.14) specifying a quality score of Q30. Trimmed reads were further processed using CLC Genomics Workbench Version 11. Paired-end reads were merged, primer locations were trimmed, and processed reads were aligned to the SacCer3 reference genome. Variants were then called using the CLC low frequency variant caller with required significance of $0.01 \%$. Variant files were exported from CLC as VCF files and downstream analysis was performed in RStudio (version 1.2.1335), paired with custom python scripting (Fig. 1). 
The variant classes included 6 possible single nucleotide variants (SNVs), single base $\mathrm{A} / \mathrm{T}$ or $\mathrm{G} / \mathrm{C}$ insertions and deletions, complex insertions and deletions, as well as mononucleotide variants (MNVs) and replacements (Replac.). MNVs are dinucleotide SNVs, where two neighboring nucleotides are both mutated, ex: CC> AT. Replacements are complex insertions or deletions, where the deleted or replaced base is a variant. Two examples include $\mathrm{AAC}>\mathrm{G}$ and $\mathrm{C}>\mathrm{AT}$. Both MNVs and replacements are extremely low frequency events and rarely occurred in our data set; neither had a significant impact on clustering. Our initial analysis assessed the frequency of each variant type as a function of genotype.

\section{Mutation Spectra Visualization}

Variants across biological replicates were analyzed in two different ways. The first, was a more conservative approach based on presence or absence of a variant at a particular position within canl for a given genotype, referred to as "unique counts". Each position-specific, unique variant was counted only once per replicate and scored based on how many biological replicates it occurred in. In our NGS approach, we cannot easily distinguish between a mutation becoming fixed early in the growth of the culture and a mutation occurring independently multiple times. By not considering the variant frequency in this analysis, we eliminated this concern. It also mitigated the effects of any "jackpot” mutations that might skew variant frequencies. The second approach incorporated the frequency of each unique variant across CAN1 (sum of frequencies). This approach added the sum of the variant frequencies if a particular variant occurred in multiple biological replicates. This analysis generated a genotype-specific mutation profile that incorporates variant type, frequency and position. Overall, the results from both types of analysis were consistent when mutation spectra were visualized. 


\section{Permissive variant analysis and filtering}

We applied a filter based on our permissive samples (RNR1, rnr1D57N, rnr1Y285F, rnrlY285A grown in the absence of selection) to remove background mutations from $\mathrm{Can}^{\mathrm{R}}$ samples. The permissive filter removes any variant that occurs below the average permissive sample unique variant frequency of $0.109 \%$ (Fig. 3C). We customized the permissive filter because we also observed position-specific variants at a frequency higher than the overall average permissive variant frequency of $0.109 \%$. Thus, these systematically higher frequency variants were removed from the selected samples if they occurred at a frequency below the highest frequency variant that occurred at the same position in the permissive data set. Fig. 3E-G shows an example of the permissive filter applied. This is a conservative filter, and undoubtedly low frequency events that are biologically relevant may be removed but are below the sensitivity of this assay. The filtering parameters can be adjusted accordingly, for other applications of this targeted sequencing approach.

\section{Determining SNV in trinucleotide context}

The trinucleotide context surrounding the SNV was determined by taking a 3 bp window surrounding the reference position within $C A N 1$. We cannot determine which strand incurred a mutation and therefore all SNVs were categorized as $\mathrm{C}$ or $\mathrm{T}$ changes for this analysis. There are a total of 96 different possible SNV changes in unique sequence context (ALEXANDROV et al. 2015). For a given sample, the number of SNVs in each of these 96 contexts was totaled. This analysis does not take frequency into account and only scores the presence or absence of a particular type of SNV. The frequency plotted is calculated based on unique counts of an SNV 
that occurred, divided by the total number of times that SNV in tri-context occurred. The data was further condensed by taking the average of each of the 96 different contexts for all the biological replicates in one genotype.

The number of trinucleotide sequence contexts in CAN1 was calculated using a sliding window approach utilizing python scripting. For each of the 96 different SNV changes in triplet context, the average number of SNVs in a genotype was divided by the number of times the triplet sequence context occurs in CAN1. This dataset was imported into R-studio and plotted via the barplot() function. The average number of times an SNV is seen in a genotype out of the total number of times the sequence context occurs across CAN1 is plotted (Fig. 5).

\section{Hierarchical cluster analysis and motif enrichment}

To identify genotype-specific variants and mutation profiles and to eliminate frequency bias from variants that occurred early on in the growth of a particular sample, we condensed unique variants based on the number of biological replicates sequenced for that genotype. While we were hesitant to include variant frequency in this analysis, we reasoned that observing a variant in multiple replicates increased the probability that it was specific to that genotype. If a variant occurred in 4 out of 4 biological replicates it was represented as 1, if it occurred in 3 out of 6 replicates it was represented as 0.5 . This strategy provided an unbiased way to assess the probability that a given variant was genotype-specific. These data were clustered on rows (or unique variants), after applying a row sum cutoff of $>2$ to eliminate low frequency variants that are less likely to be driving the observed differences in mutation spectra. Clustering the data based on unique variants allows us to identify different types of variants in specific sequence contexts that are potentially diagnostic for a particular genotype. We performed motif 
enrichment on the different variant classes (i.e. G/C deletion, CG>AT SNVs) independently, with a 12 base window surrounding the variant. Heatmaps were plotted using the pheatmap library in RStudio and motif enrichment was performed using Berkely web logos (CROOKS et al. 2004).

All data and reagents are available upon request. All variant sequence are provided in Table S5.

\section{$\underline{\text { RESULTS }}$}

\section{Rates of canavanine resistance in different genetic backgrounds}

To characterize and compare mutation profiles between different isogenetic backgrounds, we genetically altered dNTP pools using previously characterized mutations in $R N R l$, the large subunit of RNR. We chose RNR because alterations in dNTP pool levels generate distinct mutation profiles and RNR is well-associated with cancer(XU et al. 2008; KUMAR et al. 2010; KUMAR et al. 2011; BUCKLAND et al. 2014; AYE et al. 2015; MATHEWs 2015; WATT et al. 2016; MATHEWS 2018). The rnrlD57N mutation, in the activity site, leads to a balanced two-fold increase in each of the four dNTPs (CHABES et al. 2003). Two mutations in the RNR specificity site, $r n r 1 Y 285 F$ and $r n r 1 Y 285 A$, lead to three- and twenty-fold skewed increases in dCTP and dTTP, respectively (KUMAR et al. 2011). The previously characterized rnrlY285F and rnrlY285A strains also encoded a wild-type copy of $R N R 1$ under control of the inducible $p G A L 1$ promoter ( $p G A L-R N R 1)$ (KUMAR et al. 2010). Therefore, we constructed strains that encoded only $r n r 1 Y 285 F$ and $r n r 1 Y 285 A$ for comparison and for use in this study (Table S1).

We determined mutation rates of all strains at the CAN1 locus using a canavanine resistance assay (see Materials and Methods). The rnrlD57N and rnrlY285F strains exhibited 
low mutation rates (2-4 fold increase), while the $r n r 1 Y 285 A$ strain exhibited a larger $\sim 10$-fold increase in mutation rate(KUMAR et al. 2010)-(Table S5), consistent with previous work (CHABes et al. 2003; XU et al. 2008; KUMAR et al. 2010; KUMAR et al. 2011). The pGAL-RNRl construct did not affect mutation rates in $\operatorname{rnr} 1 Y 285 \mathrm{~F}$, but did modulate levels of mutagenesis in the $\operatorname{rnr} 1$ Y285A background (Table S5).

\section{Selection at $C A N 1$ paired with next generation sequencing (NGS) to define mutation profiles}

To generate mutation sequence data in RNR1/rnrl backgrounds, we selected $~ 2,000$ canavanine resistant colonies ("selected samples"), each of which sustained at least one mutation within CAN1. Resistant colonies were pooled, genomic DNA was extracted and canl was amplified in 6 overlapping regions. To amplify the 5' end of CAN1/can1, which includes a highly repetitive promoter element, we used a primer that annealed to that element, to avoid replication slippage during PCR or sequencing (Table S2: CAN1 reg1 Forward_anchored). Amplicons from each pooled sample, representing a single replicate for a given genotype, were barcoded, combined and sequenced using 2x300 paired-end sequencing (see Materials and Methods) (Fig. 1A).

Identifying genotype-specific trends in mutation profiles is complicated by the stochastic nature of mutations. To help account for this, we sequenced at least four independent samples for each genotype, using at least two independently generated isolates (Table S1 \& S4). In parallel, we sequenced pooled samples $(\sim 1,000$ colonies each) grown in the absence of canavanine selection, which we dubbed "permissive" samples (Figure 1A). These permissive controls allowed us to develop a threshold for background mutations at each position along 
CAN1 that are a result of low frequency, stochastic mutations and sequencing and/or PCR polymerase bias (see below).

We developed a custom bioinformatic pipeline for analysis. Upstream analysis was performed in CLC Genomics Workbench 11, determining variants utilizing a low frequency variant caller, and downstream analysis was performed in R Studio and through custom python scripts to determine the effect of sequence context and to compare genotypes (Fig. 1B; see Materials and Methods and below for details). All sequence data were scored for: 1) single nucleotide variants (SNVs), 2) single base (A/T or G/C) insertions or deletions, 3) complex (>1 base) insertions or deletions, 4) dinucleotide SNVs at adjacent nucleotides, i.e. mononucleotide variants (MNVs), and 5) complex replacements (see Materials and Methods). We could not distinguish which strand incurred a mutation; a $\mathrm{C}$ to A transition could also be a $\mathrm{G}$ to $\mathrm{T}$ change and was represented as such, i.e., CG> AT. NGS allowed deep sequencing of pooled samples at each position along can1, providing: 1) large sample sizes for each pooled group, 2) sequencing depth sufficient to uncover low frequency variants and 3) novel insight into mutation profiles and positional effects on mutations, all of which would be unattainable via a whole genome approach because the sequencing depth/coverage is insufficient for this type of analysis.

On average $C A N 1$ was sequenced at a depth of $16,000 x$ coverage per sample. The total variant frequency for each sample was calculated by taking the sum of the number of variants and dividing by the total number of reads sequenced for that sample. The variant frequencies for all biological replicates within a genotype were averaged. The total variant frequency for all selected samples ranged from $\sim 80 \%$ to $>100 \%$, with an average of $99.35 \%$ (Fig. 2A).

Frequencies above $100 \%$ indicated more than 1 mutation within canl in a $\mathrm{Can}^{\mathrm{R}}$ colony, and were observed in $r n r 1 Y 285 A$ strains, which had higher mutation rates (Table S5). 


\section{Permissive sample filtering removes background mutations}

Under permissive conditions (no canavanine selection), the average variant frequency for all four genetic backgrounds (wildtype, $r n r 1 D 57 N$, $r n r 1 Y 285 F$ and $r n r 1 Y 285 A$ ) was $8.7 \%$ compared to $>90 \%$ in the samples selected in the presence of canavanine (Fig. 2A). While the lower variant frequencies in permissive controls were expected, it was higher than predicted given the known mutation rates of replicative polymerases, (BEBENEK et al. 1992; KUNKEL 1992; LEE et al. 2016) indicating that these background mutations were being introduced as part of the $\mathrm{PCR} /$ sequencing pipeline. Consistent with this prediction, the variant distribution in permissive controls from wildtype, $r n r 1 D 57 N$, $r n r 1 Y 285 F$ and $r n r 1 Y 285 A$ were virtually identical, despite differences in mutation rates and mutation spectra in selected samples (CHABES et al. 2003; XU et al. 2008) (Table S5, Fig. 3A \& 3B). Of the 296 unique variants observed in all permissive samples, $82(27.7 \%)$ were observed in all 4 genotypes; over half were observed in at least 2 genetic backgrounds. Moreover, the single nucleotide variant (SNV) spectra from permissive samples, independent of genotype, (Fig. 3A) closely resembled the SNV spectrum observed for KAPA HiFi polymerase (OYOLA et al. 2012; POTAPOV AND ONG 2017), with a large bias toward $\mathrm{CG}>\mathrm{TA}$ changes.

To correct for these effects, we developed a permissive variant filter to remove potentially artefactual variants as part of the analytical pipeline. We determined the average frequency of each variant at each position along CAN1/canl observed in permissive samples, which was $0.109 \%$ compared to $0.407 \%$ in the selected samples (Fig. 3C \& 3D). Any variant that occurred below the average permissive variant frequency of $0.109 \%$ was removed by the permissive filter, which represents $\sim 1$ variant called for every 1,000 reads sequenced. Some 
position-specific variants systematically occurred at average frequencies above $0.109 \%$, in multiple biological replicates and genotypes. We incorporated this into the permissive filter, setting the threshold for each position-specific variant to its highest observed frequency in a permissive sample.

This conservative approach ensured that our analysis of mutation profiles in selected samples (see below) was not driven by background variants. We set a blanket cutoff at $0.109 \%$, rather than making the filter exclusively position-specific, because we sequenced significantly fewer permissive samples (18) than selected (47). Therefore, the permissive filter likely underestimates stochastic mutations. The higher the number of samples sequenced, the greater the probability that low frequency mutations due to "noise" will be sequenced. Fig. 3E illustrates the application of this filter, where the filter cutoff is $0.109 \%$, except for positions 31658 and 31659, where variants occurred at position-specific higher average frequencies in the permissive samples. This reduced the noise in the selected data (Fig. 3F, G), decreased variant frequency in selected samples by an average of $7.5 \%$ (Fig. 2B) and resulted in only minor changes to mutation spectra overall (Fig. S1).

\section{Alterations in dNTP pools change mutation profiles}

We determined the mutation profiles for each individual replicate (Fig. S2) and for each genotype by combining biological replicates (Fig. 4). We compared the relative levels of transitions, transversions, in/dels and other variants in our datasets with similar data from previous analyses of $r n r 1 D 57 N$, rnrlY285F pGAL-RNRI and rnrlY285A pGAL-RNRI (XU et al. 2008; KumAR et al. 2011) (Fig. S3). The distributions for the same genotypes were quite similar, but because of the depth of sequencing in our data, we were able to perform a more nuanced 
analysis (see below). The most pronounced differences were observed in the rnr1D57N spectra, likely a result of the small sample size $(\mathrm{n}=16$ canl colonies) used in the published results $[\mathrm{Xu}$ $2008]$.

After the initial analysis, we characterized mutation events in two ways: 1) the presence of a particular variant at a unique position, i.e., the number of different $\mathrm{C}>\mathrm{A}$ changes within canl ("unique variants") (Counts in Table S6) and 2) the frequency at which each of these unique variants occurred, i.e., the combined frequency of all C>A observed changes along canl ("sum of frequencies") (Freq. in Table S6) (see Materials and Methods). The latter essentially provides mutation spectra but is structured to incorporate position information. The former prevented potential "jackpot" mutations from dominating and skewing the mutation spectra. These analyses allowed us to determine whether different types of mutations occurred in a genotype-dependent manner, independent of frequency, and whether variant frequencies were altered in a significant way (Counts/Freq. in Table S6). For example, a decreased number for "unique variants" combined with unchanged or increased "sum of frequencies" indicated that variant type is more localized, possibly indicating a mutational hotspot (Table S6). Independent biological replicates with the same genotype closely resembled each other (Fig. S2); variant frequencies were more variable than unique counts among biological replicates of the same genotype (Fig. S2), consistent with the stochastic nature of mutation accumulation during the growth of a culture. Distinct mutagenic events were observed in multiple, independent, experiments, consistent with systematic, genotype-specific changes in mutation profiles.

The relative frequency of SNVs, insertions and deletions, normalized by the number of sequence reads, varied significantly by RNR1/rnrl allele (Table S6, Fig. 4), as did the absolute variant frequencies (Fig. 4B). In $r n r 1 D 57 N$, deletions increased substantially compared to 
$R N R 1$, while insertions remained unchanged. The SNV profile of $r n r 1 D 57 N$ was very similar to wildtype, although the overall SNV frequency was reduced (Table S6, Fig. 4A, B), consistent with more stochastic rather than systematic mutation events. In contrast, SNVs dominated the rnr1Y285F profile. Despite its lower mutation rate (Table S5), the normalized variant profiles of rnrlY285F were very similar to those of $\operatorname{rnr} 1 Y 285 A$, although CG>GC changes were essentially eliminated in rnr1Y285A. The proportion of variant type was skewed toward SNVs in rnrlY285F and deletions in $r n r 1 Y 285 A$. While the $r n r 1 Y 285 A$ and $r n r 1 Y 285 A$ pGAL-RNR1 strains resulted in almost indistinguishable mutation profiles, $r n r 1 Y 285 F$ and $r n r 1 Y 285 F$ pGALRNR1 showed more variation compared to one another (Table S6, Fig. S2), likely because there is a higher proportion of stochastic versus systematic mutations when mutation rates are lower. All three $r n r l$ backgrounds exhibited a significant increase in $\mathrm{G} / \mathrm{C}-1$ bp deletions compared to wild-type, although the absolute frequency varied (Table S6, Fig. 4C, D). Overall, very few insertions were observed, but we noted that the $\mathrm{G} / \mathrm{C}+1$ bp insertions were extremely rare in rnrlY285A cells compared to other genotypes (Table S6, Fig. 4E, F). The depth of sequencing coverage in the current study revealed more distinct and detailed mutation profiles than previously identified in $r n r 1 D 57 N$ and $r n r 1 Y 285 F$ (XU et al. 2008; KUMAR et al. 2010; KUMAR et al. 2011; BUCKLAND et al. 2014; WATT et al. 2016), with clear shifts in the types and frequency of mutations that accumulate in the presence of balanced versus skewed elevations in dNTP levels.

\section{Unique variants occur within $C A N 1$ in a genotype-specific manner}

Mutations occurred across the $1,772 \mathrm{bp} C A N 1$ in all genotypes tested. For each genotype, we identified unique variants in each replicate and then calculated the average variant 
frequency of each unique variant (Fig. 5, Fig. S4). Combined, we identified 860 unique variants in all genotypes tested (Fig. 5); 288 in rnrlY285A genotypes, 570 in $r n r 1 Y 285 F$ genotypes, 452 in wildtype and 322 in $\operatorname{rrn} 1 D 57 N$ (Fig. S4). On average this is over 5 times greater than the number of unique mutational events picked up by previous Sanger sequencing approaches.

Many unique variants were observed in a single isolate at low frequency, while others occurred in multiple biological replicates at increased variant frequency. The majority of average variant frequencies were below 5\%; 44/860 variants occurred at an average frequency greater than $5 \%$ (Fig. S5). When a unique variant occurred at a frequency above $25 \%$ in only one biological replicate, we defined it as a "jackpot" mutation; a mutation that arose after two generations of growth would result in $>25 \%$ of the cells (colonies) harboring that mutation. This is distinct from high frequency unique variants, which had an average frequency above 5\%, which we analyzed to identify systematic, genotype-specific variants.

We compared the average variant frequency of high frequency variants $(>5 \%$ average frequency) that were enriched within a particular genotype (Fig. 6). In $R N R 1$ (9 biological replicates), we identified 15 unique high frequency variants, 9 of which occurred in more than one $R N R 1$ biological replicate (Fig. 6A). The majority occurred in a small proportion of biological replicates (Fig. 6A). When a high frequency variant was present in multiple RNRI replicates, it typically occurred at variant frequencies of less than $1 \%$ for the remaining replicates. Most of the $R N R 1$ high frequency variants were not observed in any of the $r n r l$ backgrounds. The exception of the SNV at position 32114 which was observed in all four rnrlY285F/A genotypes. This was also the most significantly mutated position in $R N R 1$; it was in over half the biological replicates. 
In $\operatorname{rn} 1 D 57 N, 3$ high frequency unique variants were systematically mutated in multiple rnr1D57N biological replicates, and were specific to $\operatorname{rnr} 1 D 57 N$ (Fig. 6B, orange bars). For example, the $\mathrm{G}$ deletion at position 31971 occurred in 4/7 biological replicates (Fig. 6B) and drives the observed overall mutation spectrum for this genotype (Fig. 4). Similarly, $r n r 1 Y 285 F$ (periwinkle) and $\operatorname{rnr} 1 Y 285 F$ pGAL-RNRl (pink bars) exhibited very few high frequency unique variants. There was little overlap between these positions (Fig. 6C \& 6D) between the two rnrlY285F strains - or with any other genetic backgrounds. In contrast, we observed overlapping, systematic high frequency unique variants in multiple replicates of $r n r 1 Y 285 \mathrm{~A}$ (green) and $r n r 1 Y 285 A p G A L-R N R 1$ (turquoise) (Fig. 6E \& 6F), including CG>AT and GC>TA SNVs as well as G/C single base deletions. Notably, several of these high frequency unique variants were also observed in both $r n r 1 Y 285 F$ genotypes (periwinkle and pink bars) at lower frequencies, but in multiple biological replicates. This indicates that these types of mutations occur in the same sequence contexts when dCTP and dTTP levels are skewed, whether by a modest 3-fold or the more significant 20-fold increase. Variants at these positions, commonly G/C -1 deletions and CG>AT changes, are increasingly probable when dNTP pools are further elevated and skewed.

The high frequency unique variants that we observed in $r n r 1 Y 285 A-p G A L-R N R 1$ and rnr1Y285A overlapped significantly with previously defined "hotspots" in rnrlY285A pGALRNRl by Sanger sequencing (KUMAR et al. 2011; BUCKLAND et al. 2014). Our targeted sequencing approach allowed us to identify additional, previously unobserved positions in CAN1 that were highly susceptible to mutation in the $\operatorname{rrn} 1$ Y $285 \mathrm{~A}$ backgrounds, including the unique G/C deletion at position 32658 and the GC>TA SNV at position 32027 and the CG>AT SNV at 
position 32917 (Fig. 6E \& 6F). These three new high frequency unique variants each occurred in all $r n r 1 Y 285 A$ biological replicates and may be diagnostic of this genotype.

\section{Low frequency variant analysis identifies new rnr1Y285A hotspots}

The sequencing depth across canl achieved in our targeted NGS approach also allowed us to analyze low frequency variants for systematic, genotype-specific changes. For this analysis, the inclusion of multiple biological replicates was critical. We analyzed low frequency variants, defined as less than 5\% average variant frequency enriched in a genotype-specific manner. $R N R 1$, rnrlD57N and $r n r 1 Y 285 F$ mutation profiles exhibited a large number of low frequency variants (56-123) found in only one replicate and/or at low variant frequency, consistent with a high proportion of stochastic mutations. In contrast, both $r n r 1 Y 285 \mathrm{~A}$ genotypes exhibited a much smaller number of unique low frequency events (13 deletions, 16 SNVs; Fig. 7). The concentration of low frequency events in rnrlY285A backgrounds indicates a more systematic, rather than stochastic, pattern of mutagenesis. Notably, there were many G/C deletions (Fig. 7A) and CG>AT SNVs (Fig. 7B) unique to, and therefore diagnostic of, rnrlY285A backgrounds. Furthermore, these genotype-specific mutations appeared to drive the mutation profiles of rnr1Y285A backgrounds (Fig. 4). We further investigated these variants by systematically analyzing sequence context.

\section{SNVs in trinucleotide context reveal unique mutation signatures}

The analysis of both high and low frequency variants above indicated specific positions within canl that were susceptible to mutation in a genotype-specific manner, but the analysis is cumbersome and somewhat biased by frequency. The significant sequencing depth achieved in 
our study provided the opportunity to analyze genotype-specific variants and sequence context more systematically. We took two distinct approaches, which have not been previously applied to these $r n r 1$ alleles: 1) trinucleotide context analyses and 2) hierarchical cluster analyses paired with motif enrichment.

Assessing the trinucleotide context of mutations is an increasingly common approach to extract mutation signatures, especially in human cancers (ALEXANDROV et al. 2016;

HARADHVALA et al. 2018). For our analysis, each unique SNV was categorized with respect to the nucleotide immediately 5' and 3' to the variant, with 96 possible triplet contexts. We determined the average number of times an SNV was observed in a particular triplet context per genotype, normalized to the number of times the triplet context occurs in CAN1 (Fig. 8). Pearson correlation coefficients of each SNV in unique trinucleotide context were calculated to evaluate patterns of SNV mutagenesis (Fig S6 \& Table S7). Notably, in all genotypes, $\mathrm{C} \rightarrow \mathrm{T}$ changes (red bars, Fig. 8), particularly in GCC and GCG sequence contexts, dominated. The proportion of GCC and GCG changes increased with altered dNTPs, most dramatically in rnrlY285F and rnr1Y285A samples, which were highly correlated within a genotype (rnr1Y285A:rnr1Y285A $p G A L-R N R 1 \mathrm{r}_{\mathrm{s}}=0.930$, rnrlY285F:rnrlY285F pGAL-RNR1 $\left.\mathrm{r}_{\mathrm{s}}=0.947\right)$ and between $r n r 1 Y 285 F$ and $r n r 1 Y 285 A$ genotypes (rnr1Y285F:rnrlY285A $\mathrm{r}_{\mathrm{s}}=0.924, \operatorname{rnr} 1 Y 285 F$ pGALRNR1:rnr1Y285A pGAL-RNR1 $\left.\mathrm{r}_{\mathrm{s}}=0.909\right)$. RNR1 and rnrlY285A genotypes showed the weakest correlations $\left(R N R 1: \operatorname{rnr} 1 Y 285 A \mathrm{r}_{\mathrm{s}}=0.693\right.$, RNR1:rnr1Y285A pGAL-RNR1 $\left.\mathrm{r}_{\mathrm{s}}=0.658\right)$, with many variants absent in $r n r 1 Y 285 A(\mathrm{C}>\mathrm{G}$ (black), $\mathrm{T}>\mathrm{A}$ (gray), $\mathrm{T}>\mathrm{C}$ (green) and $\mathrm{T}>\mathrm{G}$ (pink) variants. For example, $\mathrm{C}>\mathrm{G}$ errors in ACG, ACT, CCG, GCA, GCG, GCT, TCC and TCT contexts were completely absent in both $r n r 1 Y 285 A$ genotypes. Many of these missing variants occurred in repetitive sequences, while errors in different repetitive contexts dominated in rnr1Y285A. 
$R N R 1$ and $r n r 1 D 57 N$ or $r n r 1 Y 285 F$ were more strongly correlated (RNR1:rnrlD57N $\mathrm{r}_{\mathrm{s}}$ $=0.774$, RNR $:$ :rnrlY285F $\left.\mathrm{r}_{\mathrm{s}}=0.736\right)$, consistent with more subtle differences between SNV spectra in trinucleotide context (Fig. 8). In $\operatorname{rnr} 1 D 57 N$, the most apparent difference is a loss of $\mathrm{C}>\mathrm{G}$ variants in CCA, CCC, CCG and CCT; both rnrlD57N and rnrlY285F also exhibited some variability in $\mathrm{T}>\mathrm{A}$ (grey), $\mathrm{T}>\mathrm{C}$ (green) and $\mathrm{T}>\mathrm{G}$ (pink) variants.

\section{Motif enrichment reveals CC dinucleotides are commonly mutated in $r n r 1 Y 285 F / A$}

\section{genotypes}

We performed hierarchical cluster analysis of the unique variants in our dataset to determine differences between mutation profiles and to determine which variants in $C A N 1 /$ canl were driving these differences. This analysis was paired with motif enrichment to determine broader sequence contexts prone to mutagenesis in a particular genetic background. We confirmed that $r n r l Y 285 A$ and $\operatorname{rnrlY285A} p G A L-R N R 1$ shared distinct features as these genotypes clustered together (Fig. 9), as did the rnrlY285F genotypes. Both rnrlY285F and rnrlY285A clustered away from $\operatorname{rnr} 1 D 57 N$ and $R N R 1$, which were more closely related in this analysis. We observed three main clusters of unique variants, labeled I, II and III.

In the first two clusters (Fig. 9, I and II) $R N R 1$ and $r n r 1 D 57 N$ were very similar, while rnr1Y285F/A exhibited distinct differential enrichment profiles. The variants in cluster I were more significantly overrepresented in $r n r 1 Y 285 A$ backgrounds, relative to $r n r 1 Y 285 F$. The

reverse was true of variants in cluster II. Both clusters I and II were underrepresented in RNRI and $r n r 1 D 57 N$. Within cluster II, there were several variants that were more significantly underrepresented in $r n r 1 D 57 N$ than in $R N R 1$. In contrast, the third cluster (III) did not exhibit 
clear trends, with different variants under- or over-represented in different genotypes. Combined, these data indicate genotype-specific mutagenesis.

From each of these three clusters, we performed motif enrichment on the sequence surrounding the variants and identified unique contexts differentially enriched in $\mathrm{rnrlY285F/A}$ backgrounds, consistent with the trinucleotide context data (Fig. 8) as well as high frequency (Fig. 6) and low frequency (Fig. 7) variant analysis. C>A and C>T changes in cluster I occurred at CC dinucleotides, while those in cluster III, which were underrepresented in $\mathrm{rnr} 1 Y 285 \mathrm{~F} / \mathrm{A}$, did not. This is consistent with the prediction that repetitive GC sequences are more prone to mutation in the presence of skewed elevations in dCTP and dTTP. Similarly, G/C deletions in repetitive G/C context were differentially enriched in rnrlY285A genotypes (Fig. 9, cluster II), while A/T insertions and deletions were enriched across all samples (Fig. 9, cluster III).

\section{DISCUSSION}

To build mutation profiles from first principles, we developed a CAN1 selection-based next generation sequencing approach to determine robust, information-rich, genotype-specific mutation profiles. We focused on RNR alleles; altered RNR activity is clearly implicated in reduced replication fidelity and carcinogenesis (MATHEWS 2015; MATHEWS 2018). While CAN1 is a widely used reporter gene in yeast, the application of deep-sequencing allowed streamlined sample preparation and more accurate determination of mutation spectra, by increasing both the number of colonies and biological replicates analyzed at one time. This approach allowed us to 1) identify low frequency sequence variants, 2) develop mutational fingerprints that resulted from compromised replication fidelity as a result of altered dNTP pools, and 3) identify broader 
sequence contexts significantly enriched in specific genotypes. We expanded our understanding of the impact of altered dNTP pools on mutagenesis and developed an approach that can be applied to study other genetic backgrounds or environmental exposures.

Our sequencing/analytic approach allowed us to define genotype-specific mutation profiles, including trinucleotide sequence context for mutations and broader sequence motifs surrounding mutations. Deep sequencing and analysis of only canl mutations identified mutation sequence motifs similar to those generated through WGS (WATT et al. 2016) and revealed new details for mutation profiles in genotypes with lower mutations rates. From this information, we can infer mechanisms of mutagenesis (see below). Importantly, the motifs identified previously in $r n r 1 Y 285 A$ lines also carried a $m s h 2 \Delta$, effectively eliminating MMR, which might alter the diagnostic sequence contexts in which mutations are specifically present or absent. We identified sequence motifs in $r n r 1 Y 285 A$ similar to those in $r n r 1 Y 285 A$ msh2 $\Delta$ from WGS (WATT et al. 2016), indicating that the errors generated when dNTPs are elevated and skewed are substrates for mismatch repair.

\section{Mechanisms of mutagenesis from distinct elevations in dNTP levels}

Despite a 2-fold increase in mutation rate above wildtype, the $\operatorname{rnr} 1 D 57 N$ mutation spectrum closely resembled wildtype (Fig. 4), consistent with previous studies (XU et al. 2008; KUMAR et al. 2011). This indicated that the 2-fold balanced increase in dNTPs causes errors to accumulate in a stochastic manner, similar to wildtype. Given the low mutation rate in rnrlD57N (Table S5), the majority of these errors are likely corrected by the mismatch repair (MMR) system, which identifies replication errors and targets them for repair(XU et al. 2008; KUNKEL AND ERIE 2015). Nonetheless, we observed a significant increase in G/C single base 
deletions in this genetic background (Fig. 4B) and were able to identify new sequence contexts specific to mutations in rnrlD57N. This includes CG>TA, CG>AT changes, A/T deletions in repetitive A/T sequences (Figs. 4, 6B, 9), which appeared diagnostic of rnr1D57N mutagenesis. We predict that these regions are inherently more prone to mutation; the two-fold increase in dNTP levels exacerbates this by favoring replicative polymerase synthesis over proofreading activity.

The high frequency of G/C deletions observed in $\operatorname{rnr} 1 D 57 N$ was driven by a single base $\mathrm{G}$ deletion at position 31971 that was mutated in 4 out of 7 rnr1D57N replicates and occurs at a high frequency, on average $\sim 60 \%$ (Figs. 4B \& 6B, S2D - compare unique counts vs. frequency of variants). The high frequency indicated the mutation arose early in the growth of the cultures, but the fact that it occurred and was enriched in multiple biological replicates indicated that this position is uniquely susceptible to deletion, specifically in rnrlD57N. This variant was bordered by repeats on both the 5' side (repetitive A/T motif) and 3' side (CC dinucleotide)

(ATAAGCCA). Therefore, both the excess in dTTP incorporated opposite the AA dinucleotide and the excess of dCTP incorporated on the opposite strand could result in transient misalignment and misincorporation at this position and it is therefore likely this variant occurred during both leading and lagging strand replication. Increased dNTP levels alter replication fork dynamics(DAVIDSON et al. 2012; POLI et al. 2012), potentially enhancing the next nucleotide effect and resulting in increased frequencies at this position in $\operatorname{rnrlD57N}$.

The $\operatorname{rnrlY285F}$ allele exhibited a modest effect on mutation rate, but a distinct mutation profile (Fig. 4). While the profile shift was more subtle than in rnrlY285A, meta-analysis of the types and positions of variants in $r n r 1 Y 285 F$ significantly overlapped with that of $r n r 1 Y 285 A$ (Figs. 6, 8, 9), despite the differences in mutation rate (Table S5) and dCTP/dTTP levels 
(KUMAR et al. 2010). This was particularly noticeable with $\mathrm{G} / \mathrm{C}$ variants, i.e. G/C deletions and CG>AT variants. Therefore, even modestly increased dCTP and dTTP pools resulted in distinct error accumulation. We predict an increased probability that replicative polymerases incorporated the excess nucleotides (dCTP, dTTP) during synthesis and more efficiently extended mismatches at the expense of proofreading (KUMAR et al. 2011; WATT et al. 2016).

We noted a substantial increase in $\mathrm{G} / \mathrm{C}$ deletions specific to rnr1Y285A backgrounds (Fig. 6E \& 6F), which may be explained by limiting levels of dGTP in rnrlY285A. dGTP levels are already limiting in both yeast and mammalian cells(CHABES et al. 2003; HÅKANSSON et al. 2006); in $r n r 1 Y 285 A$, the proportion of dGTP relative to the total dNTP pool is extremely small, reducing the probability of its incorporation. We predict that this led to this distinct pattern of deletion in rnrlY285F/A (MATHEWS 2015), within specific sequence contexts, i.e. CC and CCC runs (Fig. 9). Notably, rnrl alleles that dramatically increased dGTP levels were also severely mutagenic ( $r n r 1 K 243 E$ and $r n r 11262 \mathrm{~V}, \mathrm{~N} 291 D$ ), increasing SNVs and frame shift mutations in repetitive contexts(SCHMIDT et al. 2019). This highlights the importance of proper absolute and relative abundance of dGTP.

Our data indicated that the mutation profiles generated using our sequencing and analytical approach were diagnostic of the balanced or unbalanced nature of the dNTP pools, i.e. which dNTPs were elevated. While the mutation rate and variant frequencies reflected the absolute levels of dNTPs; far more in/dels than SNVs were observed for $r n r 1 Y 285 A$ while the

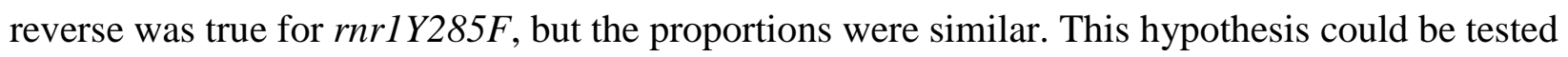
by comparing the $r n r 1 D 57 N$ mutation profile with that of $R N R 1$ galactose-induced overexpression, which similarly leads to balanced dNTP pools, but elevated $\sim 10$-fold above wildtype levels (CHABES AND STILLMAN 2007), as well as other rnrl alleles. 


\section{Implications for understanding mutation signatures in human cancers}

Mutation signatures of human tumors are used to identify molecular drivers of carcinogenesis (AlEXANDROV et al. 2013; NIK-ZAINAL et al. 2016; HARADHVALA et al. 2018; ALEXANDROV et al. 2020), which have clear implications for diagnosis, prognosis and treatment options for patients (VAN HOECK et al. 2019). Tumor mutation signatures are typically extracted from a large sampling of human tumor information via mathematical approaches; implicated molecular pathways are inferred. This has led to spurious associations between mutation profiles and driver mutations, e.g. RefSig 3 and BRCA1/BRCA2 (DEGASPERI et al. 2020). In contrast, we used the $S$. cerevisiae model system to develop information-rich mutation profiles from first principles, using multiple biological replicates of defined genetic backgrounds alongside wildtype controls.

Importantly, elevated dNTP levels (balanced or skewed) have not been considered when evaluating mutation signatures from human tumors, although they almost certainly contribute to mutagenesis in cancer (AYE et al. 2015; MATHEWs 2015; MATHEWs 2017; PAI AND KEARSEY 2017; DEGASPERI et al. 2020). We noted distinct similarities between rnrl SNV triplet mutation profiles (Fig. 8) and specific COSMIC signatures, most notably signatures 6 and 15. Signature 6 occurs most commonly in colorectal and uterine cancers and is associated with defective MMR. We previously noted synergistic effects on mutation rate between $\operatorname{rnr} 1 \mathrm{D} 57 \mathrm{~N}$ and MMR deletions(XU et al. 2008). The contribution of elevated dNTP pool levels to mutagenesis in combination with MMR is intriguing.

While elevated dNTP levels have been implicated in supporting rapidly proliferating cancer cells, it remains to be determined whether these are skewed or balanced increases, which 
could be tumor-specific (WILSON et al. 2011; KOHNKEN et al. ; MATHEWS ; PURHONEN et al. 2020). Quantification of dNTP levels in HeLa cell lines demonstrated elevated levels of all four dNTPs with an approximate 2-fold increase in dATP and dCTP and a 4-fold increase in dTTP and dGTP (MATHEWs 2015). Different skewed elevations result in distinct mutation spectra (SCHMIDT et al. 2019) and thus more studies are necessary to determine what dNTP imbalances are relevant to different types of cancers. In the meantime, certain indicator mutations, such as a high number of $\mathrm{G} / \mathrm{C}$ single base deletions, can point towards specific $\mathrm{dNTP}$ imbalances i.e. high dCTP and dTTP levels seen in $r n r l Y 285 A$.

\section{ACKNOWLEDGEMENTS}

We are grateful to Dr. Andrei Chabes for providing strains and plasmids. We are grateful to Dr. Don Yergeau for his advice in next generation sequencing. We are grateful to members of the Surtees lab (past and present) for helpful discussions and input. We are grateful to Dr. Mark Sutton and Dr. John Panepinto for input and helpful discussions.

\section{FUNDING}

N.A.L. is a University at Buffalo Presidential Scholar. This work was supported by the American Cancer Society (RSG-14-2350-01) to J.A.S. J.A.S. is an ACS Research Scholar. J.A.S. is also grateful for support from the University at Buffalo's Genome, Environment and Microbiome (GEM) Community of Excellence. Funding for open access charge: GEM. 


\section{CONFLICT OF INTEREST}

The authors have no conflicts to disclose.

\section{REFERENCES}

Alexandrov, L. B., P. H. Jones, D. C. Wedge, J. E. Sale, P. J. Campbell et al., 2015 Clock-like mutational processes in human somatic cells. Nat Genet 47: 1402-1407.

Alexandrov, L. B., Y. S. Ju, K. Haase, P. Van Loo, I. Martincorena et al., 2016 Mutational signatures associated with tobacco smoking in human cancer. Science 354: 618-622.

Alexandrov, L. B., J. Kim, N. J. Haradhvala, M. N. Huang, A. W. Tian Ng et al., 2020 The repertoire of mutational signatures in human cancer. Nature 578: 94-101.

Alexandrov, L. B., S. Nik-Zainal, D. C. Wedge, S. A. Aparicio, S. Behjati et al., 2013 Signatures of mutational processes in human cancer. Nature 500: 415-421.

Aye, Y., M. Li, M. J. Long and R. S. Weiss, 2015 Ribonucleotide reductase and cancer: biological mechanisms and targeted therapies. Oncogene 34: 2011-2021.

Bebenek, K., J. D. Roberts and T. A. Kunkel, 1992 The effects of dNTP pool imbalances on frameshift fidelity during DNA replication. J Biol Chem 267: 3589-3596.

Brash, D. E., 2015 UV signature mutations. Photochemistry and photobiology 91: 15-26.

Buckland, R. J., D. L. Watt, B. Chittoor, A. K. Nilsson, T. A. Kunkel et al., 2014 Increased and imbalanced dNTP pools symmetrically promote both leading and lagging strand replication infidelity. PLoS Genet 10: e1004846.

Chabes, A., B. Georgieva, V. Domkin, X. Zhao, R. Rothstein et al., 2003 Survival of DNA damage in yeast directly depends on increased dNTP levels allowed by relaxed feedback inhibition of ribonucleotide reductase. Cell 112: 391-401. 
Chabes, A., and B. Stillman, 2007 Constitutively high dNTP concentration inhibits cell cycle progression and the DNA damage checkpoint in yeast Saccharomyces cerevisiae. Proc Natl Acad Sci U S A 104: 1183-1188.

Chan, K., S. A. Roberts, L. J. Klimczak, J. F. Sterling, N. Saini et al., 2015 An APOBEC3A hypermutation signature is distinguishable from the signature of background mutagenesis by APOBEC3B in human cancers. Nat Genet 47: 1067-1072.

Chan, K., J. F. Sterling, S. A. Roberts, A. S. Bhagwat, M. A. Resnick et al., 2012 Base damage within single-strand DNA underlies in vivo hypermutability induced by a ubiquitous environmental agent. PLoS Genet 8: e1003149.

Crooks, G. E., G. Hon, J. M. Chandonia and S. E. Brenner, 2004 WebLogo: a sequence logo generator. Genome Res 14: 1188-1190.

Davidson, M. B., Y. Katou, A. Keszthelyi, T. L. Sing, T. Xia et al., 2012 Endogenous DNA replication stress results in expansion of dNTP pools and a mutator phenotype. Embo $\mathrm{j}$ 31: 895-907.

Degasperi, A., T. D. Amarante, J. Czarnecki, S. Shooter, X. Zou et al., 2020 A practical framework and online tool for mutational signature analyses show inter-tissue variation and driver dependencies. Nat Cancer 1: 249-263.

Fantes, P. A., and J. Creanor, 1984 Canavanine resistance and the mechanism of arginine uptake in the fission yeast Schizosaccharomyces pombe. J Gen Microbiol 130: 3265-3273.

Gietz, D., A. St Jean, R. A. Woods and R. H. Schiestl, 1992 Improved method for high efficiency transformation of intact yeast cells. Nucleic Acids Res 20: 1425. 
Håkansson, P., A. Hofer and L. Thelander, 2006 Regulation of mammalian ribonucleotide reduction and dNTP pools after DNA damage and in resting cells. J Biol Chem 281: 7834-7841.

Haradhvala, N. J., J. Kim, Y. E. Maruvka, P. Polak, D. Rosebrock et al., 2018 Distinct mutational signatures characterize concurrent loss of polymerase proofreading and mismatch repair. Nat Commun 9: 1746.

Hoffmann, W., 1985 Molecular characterization of the CAN1 locus in Saccharomyces cerevisiae. A transmembrane protein without $\mathrm{N}$-terminal hydrophobic signal sequence. $\mathrm{J}$ Biol Chem 260: 11831-11837.

Howard, B. D., and I. Tessman, 1964 IDENTIFICATION OF THE ALTERED BASES IN MUTATED SINGLE-STRANDED DNA. 3. MUTAGENESIS BY ULTRAVIOLET LIGHT. J Mol Biol 9: 372-375.

Jordheim, L. P., D. Durantel, F. Zoulim and C. Dumontet, 2013 Advances in the development of nucleoside and nucleotide analogues for cancer and viral diseases. Nat Rev Drug Discov 12: 447-464.

Kohnken, R., K. M. Kodigepalli and L. Wu, 2015 Regulation of deoxynucleotide metabolism in cancer: novel mechanisms and therapeutic implications. Mol Cancer 14: 176.

Kumar, D., A. L. Abdulovic, J. Viberg, A. K. Nilsson, T. A. Kunkel et al., 2011 Mechanisms of mutagenesis in vivo due to imbalanced dNTP pools. Nucleic Acids Res 39: 1360-1371.

Kumar, D., J. Viberg, A. K. Nilsson and A. Chabes, 2010 Highly mutagenic and severely imbalanced dNTP pools can escape detection by the S-phase checkpoint. Nucleic Acids Res 38: 3975-3983.

Kunkel, T. A., 1992 DNA replication fidelity. J Biol Chem 267: 18251-18254. 
Kunkel, T. A., and D. A. Erie, 2015 Eukaryotic Mismatch Repair in Relation to DNA Replication. Annu Rev Genet 49: 291-313.

Kunkel, T. A., and A. Soni, 1988 Mutagenesis by transient misalignment. J Biol Chem 263: 14784-14789.

Lee, D. F., J. Lu, S. Chang, J. J. Loparo and X. S. Xie, 2016 Mapping DNA polymerase errors by single-molecule sequencing. Nucleic Acids Res 44: e118.

Lujan, S. A., A. R. Clausen, A. B. Clark, H. K. MacAlpine, D. M. MacAlpine et al., 2014 Heterogeneous polymerase fidelity and mismatch repair bias genome variation and composition. Genome Res 24: 1751-1764.

Mathews, C. K., 2015 Deoxyribonucleotide metabolism, mutagenesis and cancer. Nat Rev Cancer 15: 528-539.

Mathews, C. K., 2017 Oxidized deoxyribonucleotides, mutagenesis, and cancer. Faseb j 31: 1113.

Mathews, C. K., 2018 Still the most interesting enzyme in the world. Faseb j 32: 4067-4069.

Mauney, C. H., and T. Hollis, 2018 SAMHD1: Recurring roles in cell cycle, viral restriction, cancer, and innate immunity. Autoimmunity 51: 96-110.

Nik-Zainal, S., H. Davies, J. Staaf, M. Ramakrishna, D. Glodzik et al., 2016 Landscape of somatic mutations in 560 breast cancer whole-genome sequences. Nature 534: 47-54.

Oyola, S. O., T. D. Otto, Y. Gu, G. Maslen, M. Manske et al., 2012 Optimizing illumina nextgeneration sequencing library preparation for extremely at-biased genomes. BMC Genomics 13: 1 .

Pai, C. C., and S. E. Kearsey, 2017 A Critical Balance: dNTPs and the Maintenance of Genome Stability. Genes (Basel) 8. 
Phear, G., J. Nalbantoglu and M. Meuth, 1987 Next-nucleotide effects in mutations driven by DNA precursor pool imbalances at the aprt locus of Chinese hamster ovary cells. Proc Natl Acad Sci U S A 84: 4450-4454.

Poli, J., O. Tsaponina, L. Crabbé, A. Keszthelyi, V. Pantesco et al., 2012 dNTP pools determine fork progression and origin usage under replication stress. Embo j 31: 883-894.

Potapov, V., and J. L. Ong, 2017 Examining Sources of Error in PCR by Single-Molecule Sequencing. PLoS One 12: e0169774.

Purhonen, J., R. Banerjee, A. E. McDonald, V. Fellman and J. Kallijärvi, 2020 A sensitive assay for dNTPs based on long synthetic oligonucleotides, EvaGreen dye and inhibitor-resistant high-fidelity DNA polymerase. Nucleic Acids Research 48: e87-e87.

Rentoft, M., K. Lindell, P. Tran, A. L. Chabes, R. J. Buckland et al., 2016 Heterozygous colon cancer-associated mutations of SAMHD1 have functional significance. Proc Natl Acad Sci U S A 113: 4723-4728.

Saini, N., J. F. Sterling, C. J. Sakofsky, C. K. Giacobone, L. J. Klimczak et al., 2020 Mutation signatures specific to DNA alkylating agents in yeast and cancers. Nucleic Acids Res 48: 3692-3707.

Schmidt, T. T., S. Sharma, G. X. Reyes, K. Gries, M. Gross et al., 2019 A genetic screen pinpoints ribonucleotide reductase residues that sustain dNTP homeostasis and specifies a highly mutagenic type of dNTP imbalance. Nucleic Acids Res 47: 237-252.

Sikorski, R. S., and P. Hieter, 1989 A system of shuttle vectors and yeast host strains designed for efficient manipulation of DNA in Saccharomyces cerevisiae. Genetics 122: 19-27.

Tate, J. G., S. Bamford, H. C. Jubb, Z. Sondka, D. M. Beare et al., 2018 COSMIC: the Catalogue Of Somatic Mutations In Cancer. Nucleic Acids Research 47: D941-D947. 
Van Hoeck, A., N. H. Tjoonk, R. van Boxtel and E. Cuppen, 2019 Portrait of a cancer: mutational signature analyses for cancer diagnostics. BMC Cancer 19: 457.

Watt, D. L., R. J. Buckland, S. A. Lujan, T. A. Kunkel and A. Chabes, 2016 Genome-wide analysis of the specificity and mechanisms of replication infidelity driven by imbalanced dNTP pools. Nucleic Acids Res 44: 1669-1680.

Wilson, P. M., M. J. Labonte, J. Russell, S. Louie, A. A. Ghobrial et al., 2011 A novel fluorescence-based assay for the rapid detection and quantification of cellular deoxyribonucleoside triphosphates. Nucleic Acids Res 39: e112.

Xu, X., J. L. Page, J. A. Surtees, H. Liu, S. Lagedrost et al., 2008 Broad overexpression of ribonucleotide reductase genes in mice specifically induces lung neoplasms. Cancer Res 68: $2652-2660$.

Zhu, Y. O., M. L. Siegal, D. W. Hall and D. A. Petrov, 2014 Precise estimates of mutation rate and spectrum in yeast. Proc Natl Acad Sci U S A 111: E2310-2318.

\section{Figure Legends}

Figure 1. A schematic of (A) experimental design and (B) pipeline for data analysis.

Figure 2. Absolute variant frequency is consistent with selection at $C A N 1$. The average absolute variant frequency was calculated for each genotype by dividing the total number of 
variants by the total number of reads sequenced. The average was then taken for all biological replicates sequenced in a genotype. Error bars represent the standard deviation between biological replicates within each genotype. The number of total biological replicates sequenced varied by genotype; numbers are displayed in Table S4. (A) The average total variant frequency differed significantly between selected and permissive samples, regardless of genotype. (B) The average variant frequency decreased following application of the permissive variant filter.

\section{Figure 3. Permissive controls show consistent mutation spectra and are filtered from our}

data set. (A) The SNV spectra for all permissive samples. Plotted are the number of unique variants in a sample normalized out of $100 \%$ for comparison purposes. Individual biological replicates for each genotype are shown. (B) The deletion spectra for each permissive sample. Shown are single G/C (blue), A/T (green) base deletions and deletions greater than 1 base (pink). Individual biological replicates for each genotype are shown. (C) A histogram plotting the number of variants (y-axis) and the frequency (x-axis) at which they occur in both permissive and (D) selected samples. The red line represents the average variant frequency in permissive and selected samples respectively. (E) A 100 bp window from 32,000 to 32,100 displaying the different types of variants and the frequency at which they occur in 4 different permissive genotypes. (F) The same window displaying the variants that occur in the same four genotypes where mutants were selected in the presence of canavanine. (G) Variants that remain in selected samples post-permissive variant filter.

Figure 4. Mutation spectra vary by rnr1 allele. (A) The SNV spectra normalized to total SNVs (upper panel) and normalized out of total variants (lower panel). (B) The deletion spectra 
normalized to total deletions (upper) and normalized to total variants (lower). (C) The insertion spectra normalized to total deletions (upper) and normalized out of total variants (lower).

Figure 5. Variant spread across $\boldsymbol{C A N 1}$. The average variant frequency of a unique low frequency $(<5 \%, y$-axis) variants within a genotype is plotted. High frequency variants are plotted in Figure S5. CAN1 is annotated by position on Chromosome V on the (-) strand. The transcriptional start site (TSS) and arrow show the direction of transcription from the start 5' to the end of the gene, 3 '.

Figure 6. High frequency variants occur systematically in $r n r 1 Y 285 F / A$ genotypes. Unique variants with an average variant frequency $>5 \%$ for (A) wildtype, (B) $\operatorname{rnr} 1 D 57 N,(\mathbf{C})$ rnr1Y285F-pGAL RNR1, (D) rnr1Y285F, (E) rnr1Y285A-pGAL-RNR1, (F) rnr1Y285A. For comparison, the average frequency of each variant in other genotypes is also plotted. Below each plot, the fraction of biological replicates in which the variant occurred is observed and the surrounding sequence context are indicated. *, unique variant occurred in $\geq 50 \%$ of biological replicates for the genotype analyzed;**, unique variant occurred in $100 \%$ of biological replicates for the genotype analyzed.

Figure 7. Low frequency variants specific to rnr1Y285A genotypes. Low frequency deletions (A) and SNVS (B) in rnrlY285A pGAL-RNR1 (turquoise) and rnrlY285A (green) genotypes. None of these variants were observed in wildtype, but some occurred at low frequencies in rnrlD57N (brown) and $r n r 1 Y 285 F$ (blue and pink) genotypes. *, variant occurred in $\geq 50 \%$ of 
rnr1Y285A or rnr1Y285A pGAL-RNR1 biological replicates; **, variant occurred in $100 \%$ of the rnr1Y285A or $\operatorname{rnr} 1 Y 285 A$ pGAL-RNR1 biological replicates analyzed.

Figure 8. The average number of each SNV as it occurs in unique triplet nucleotide context is distinct between rnr1 alleles. Bars are colored according to the six different types of SNVs. (A) The 16 different triplet contexts are lettered for display purposes. The variant change $(\mathrm{C}>\mathrm{A}$, turquoise bar) occurs at the middle nucleotide marked $\mathrm{X}$ in each triplet context for (B) RNRI, (C) rnr1D57N, (D) rnr1Y285F pGAL-RNR1, (E) rnr1Y285F, (F) rnr1Y285A pGAL-RNR1 and

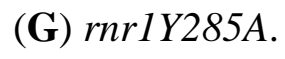

\section{Figure 9. Unique variants in enriched motifs form distinct clusters.}

(A) Hierarchical cluster analysis of the highest occurring variants reveals distinct clusters, annotated I, II and III. (B) The different classes of variants enriched in each cluster were subset, and 12 basepairs surrounding the variant was used to perform motif enrichment. The corresponding motifs from the different variant classes in each boxed cluster are displayed on the right and labeled accordingly (box I, II and III). 


\section{Selection on SC-ARG+CAN: $\sim 2,000$ Can $^{R}$ colonies

Nextera barcoding
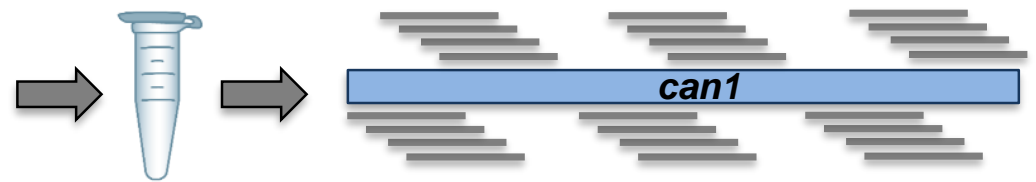

CAN1 PCR amplification with KAPA HiFi polymerase

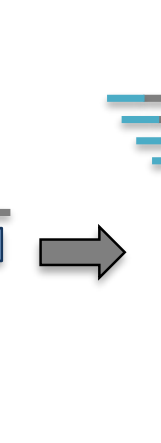

Sample 1

Index

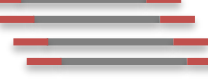

Sample 2
Illumina paired-end sequencing

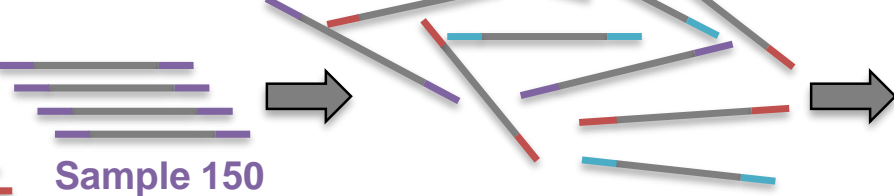

Index

Index
Library pooling
R1

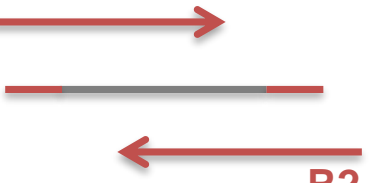

Permissive controls; 2,000

colonies grown on SC-ARG

\section{CLC Genomics} Workbench

$\mathrm{R} 1$ and $\mathrm{R} 2$
Merged

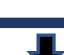

$\sqrt{2}$

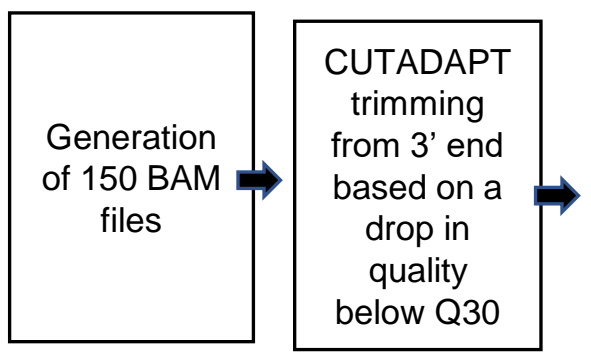

Primer trim

from 5' end

sequencing

eads into

CLC

Genomics

Workbench

\begin{tabular}{|c|}
\hline \\
\hline \\
\hline Aligned to \\
SacCer3 \\
reference
\end{tabular}$$
\text { genom }
$$

Low
Frequency

Variant

Caller

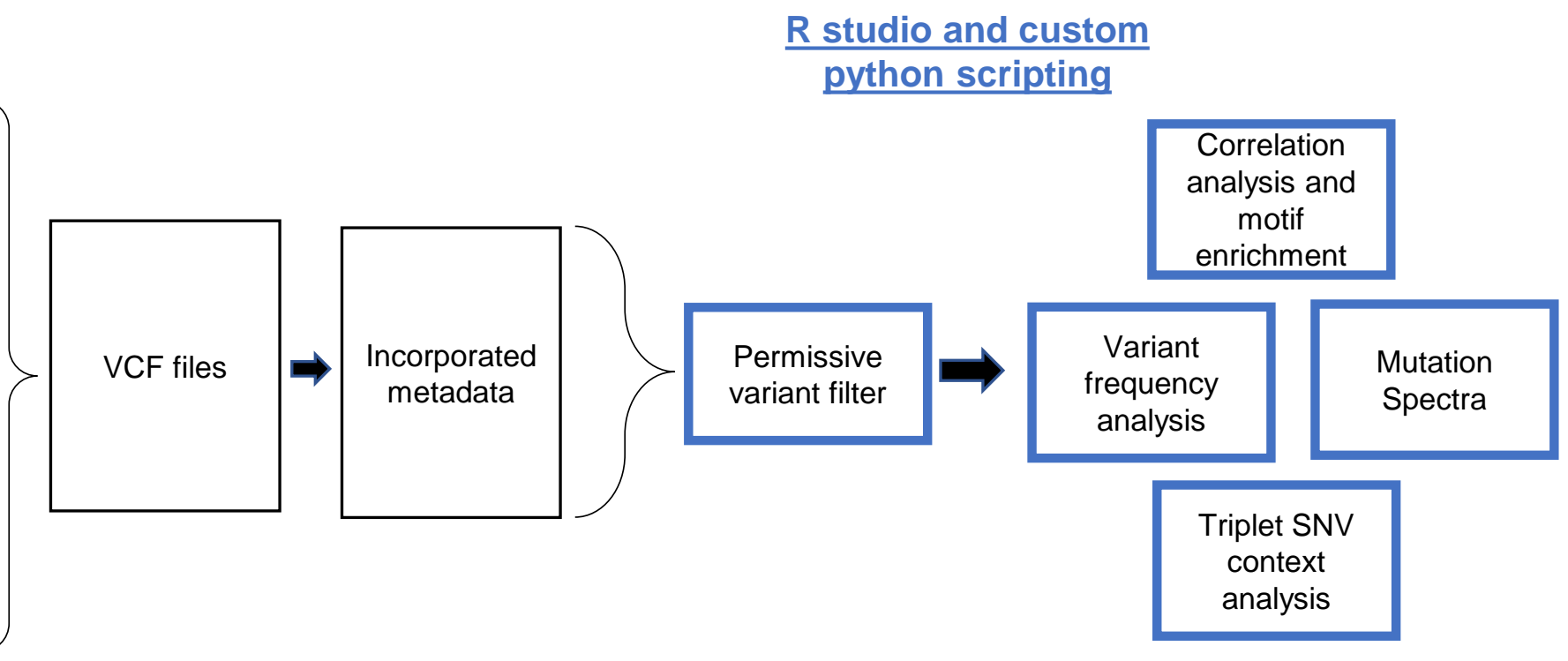

Figure 1. 
A

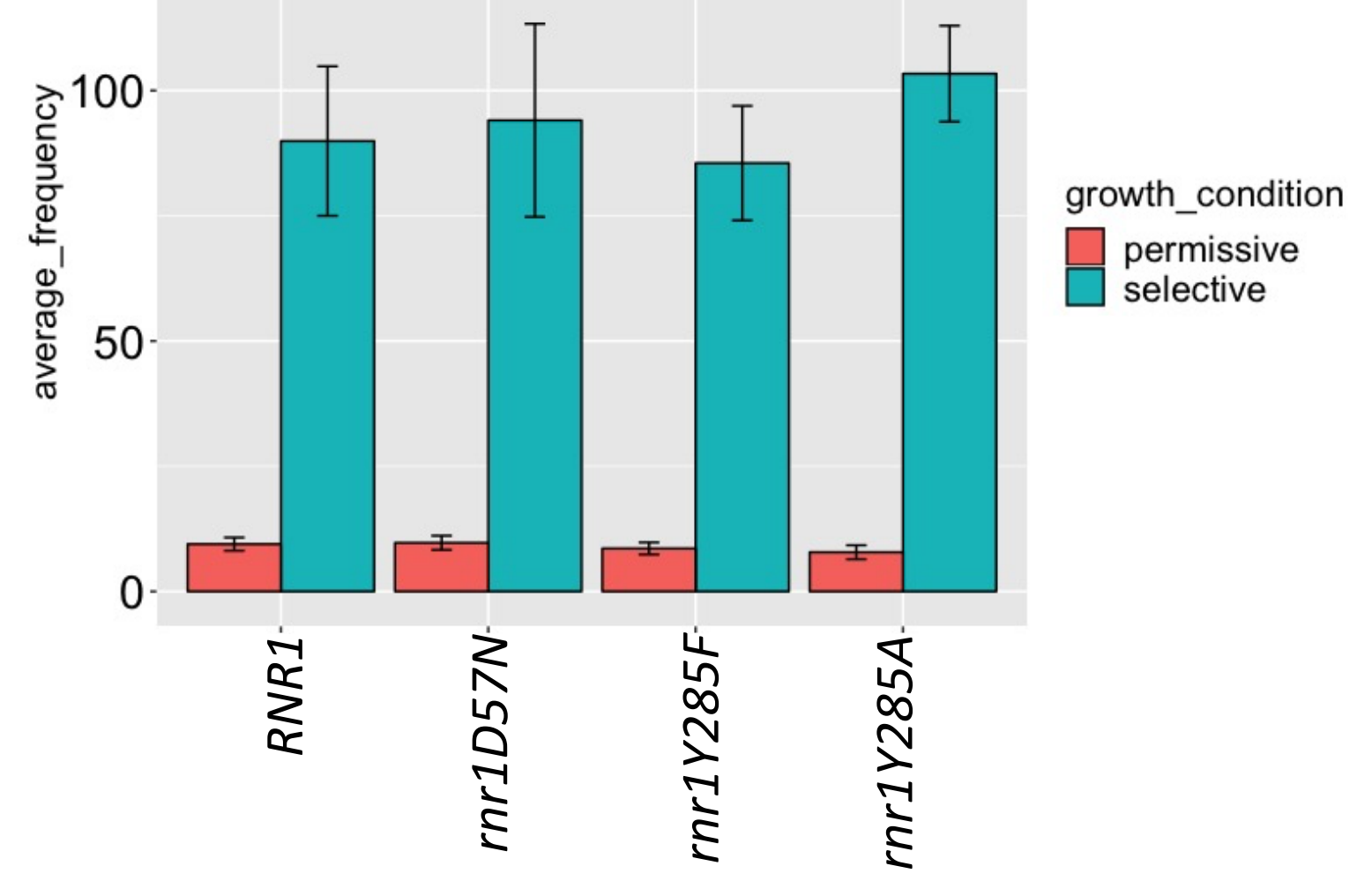

B

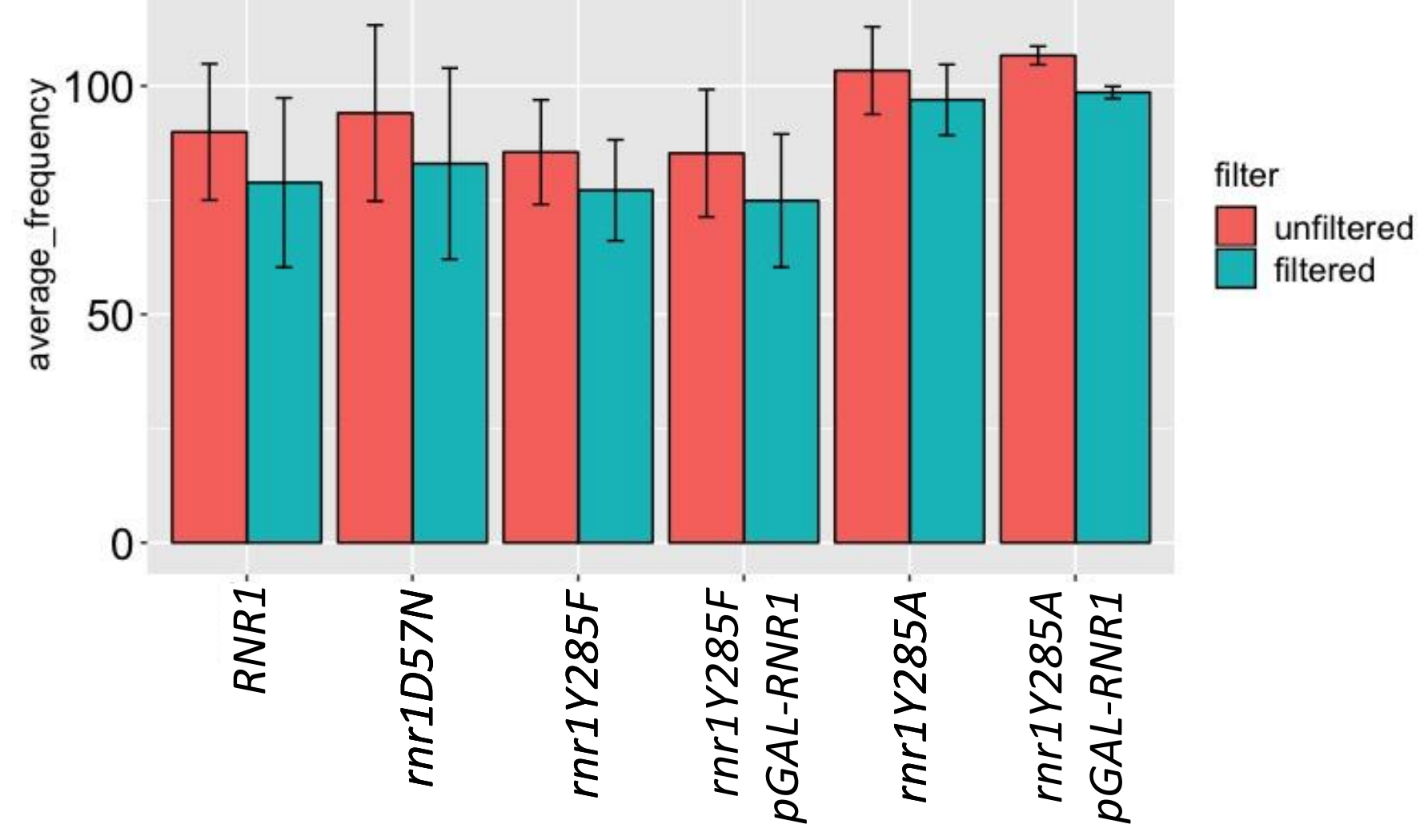



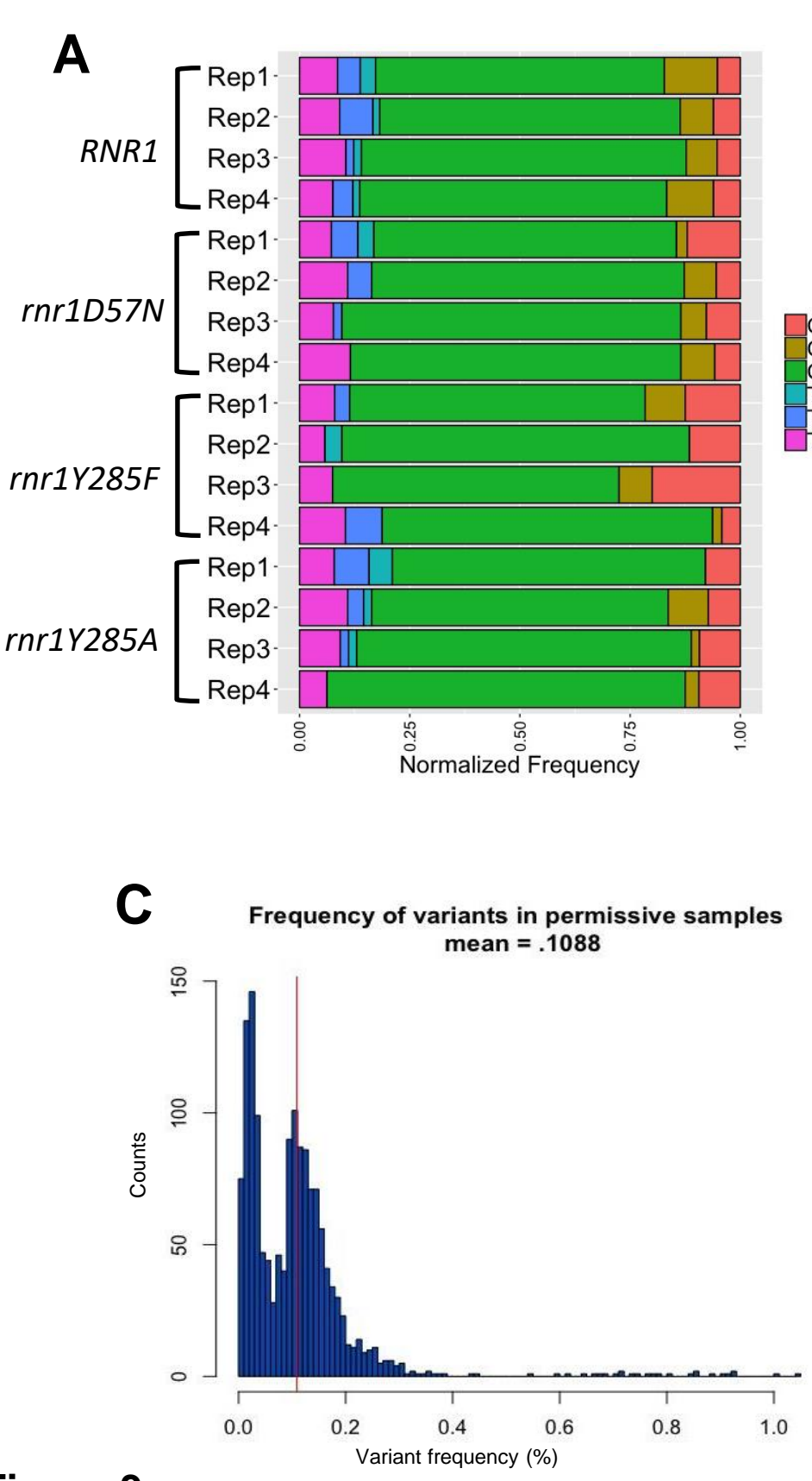

Figure 3.
B

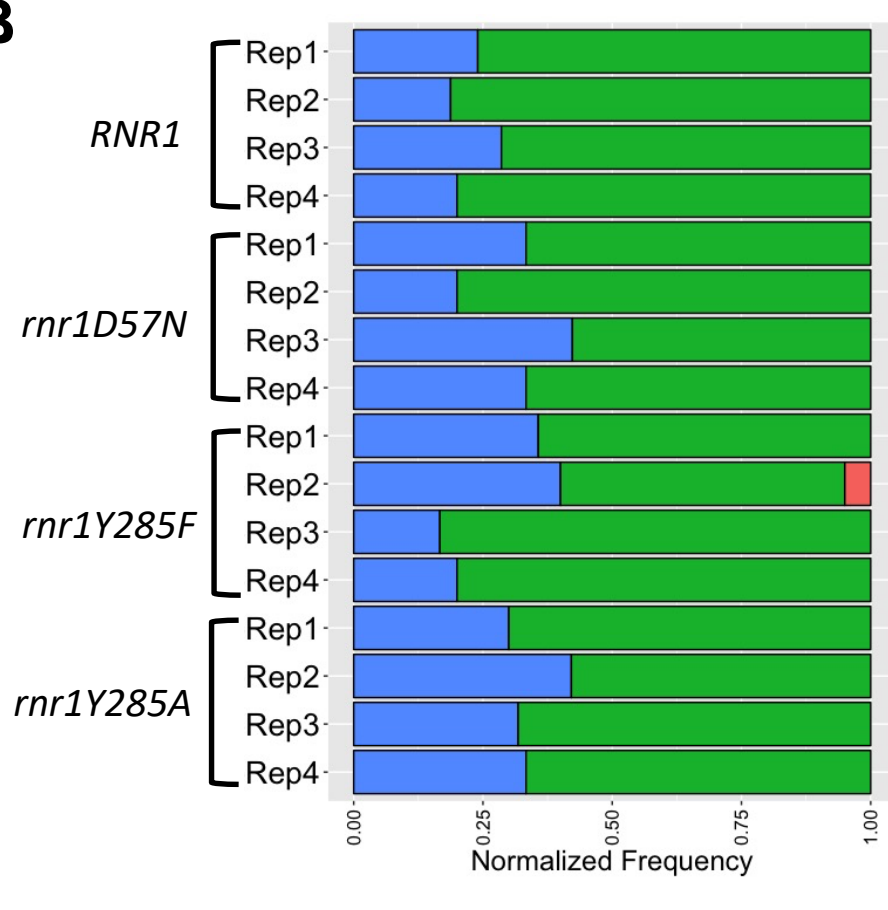

D

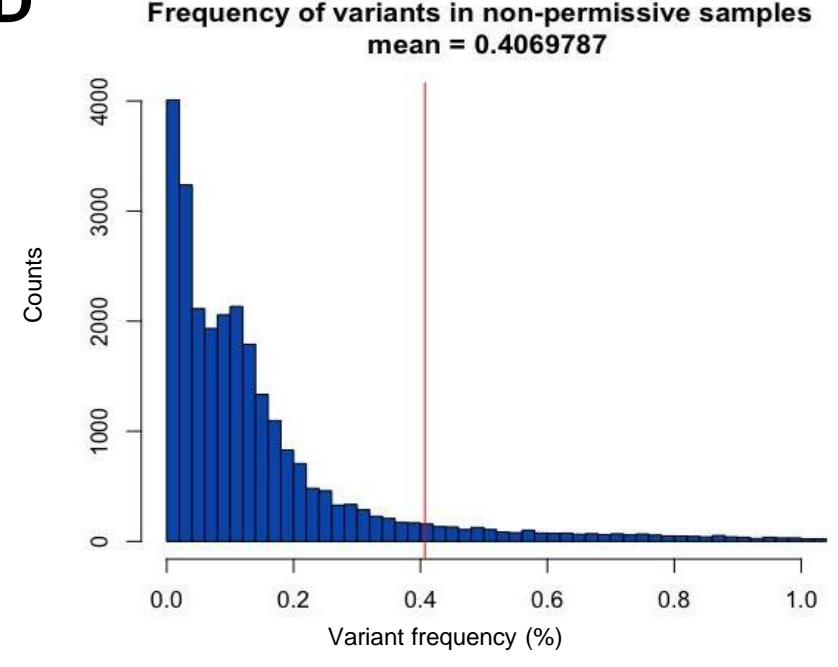

E

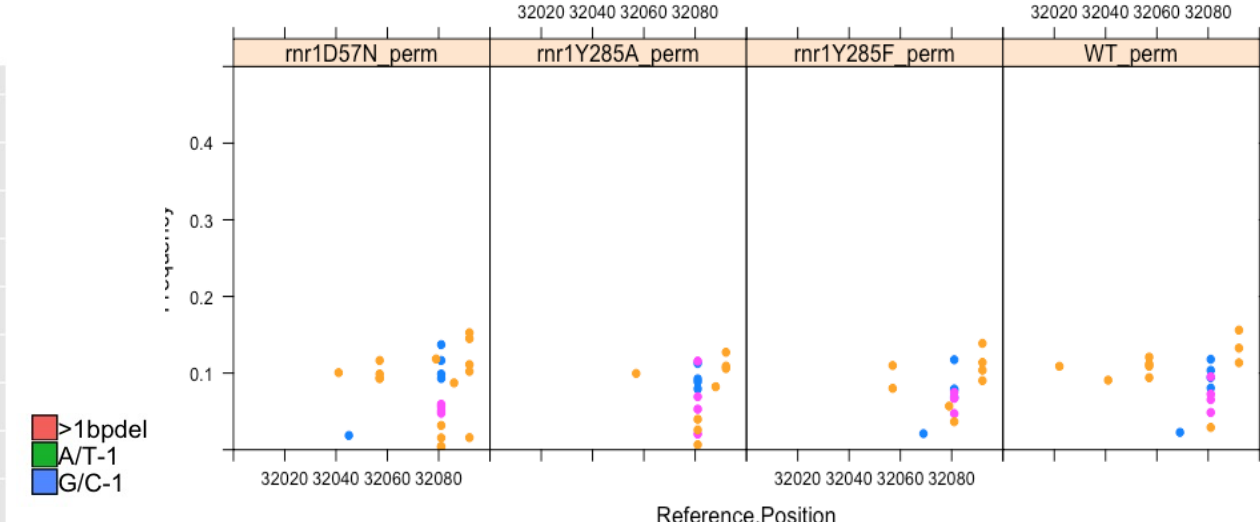

F

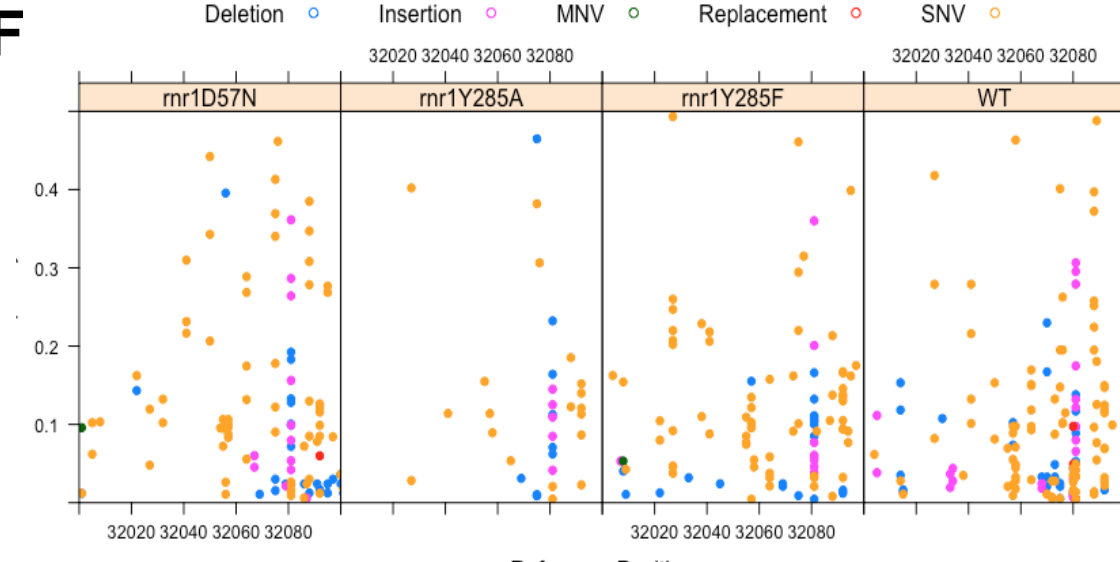

MNV ○ Replacement ○ SNV Reference.Position

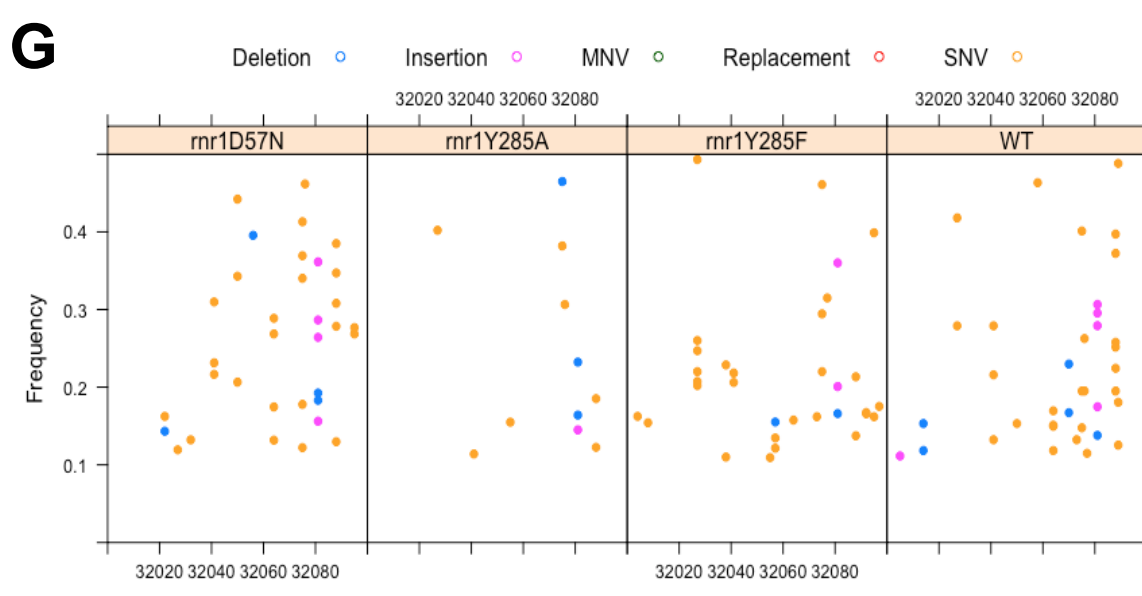




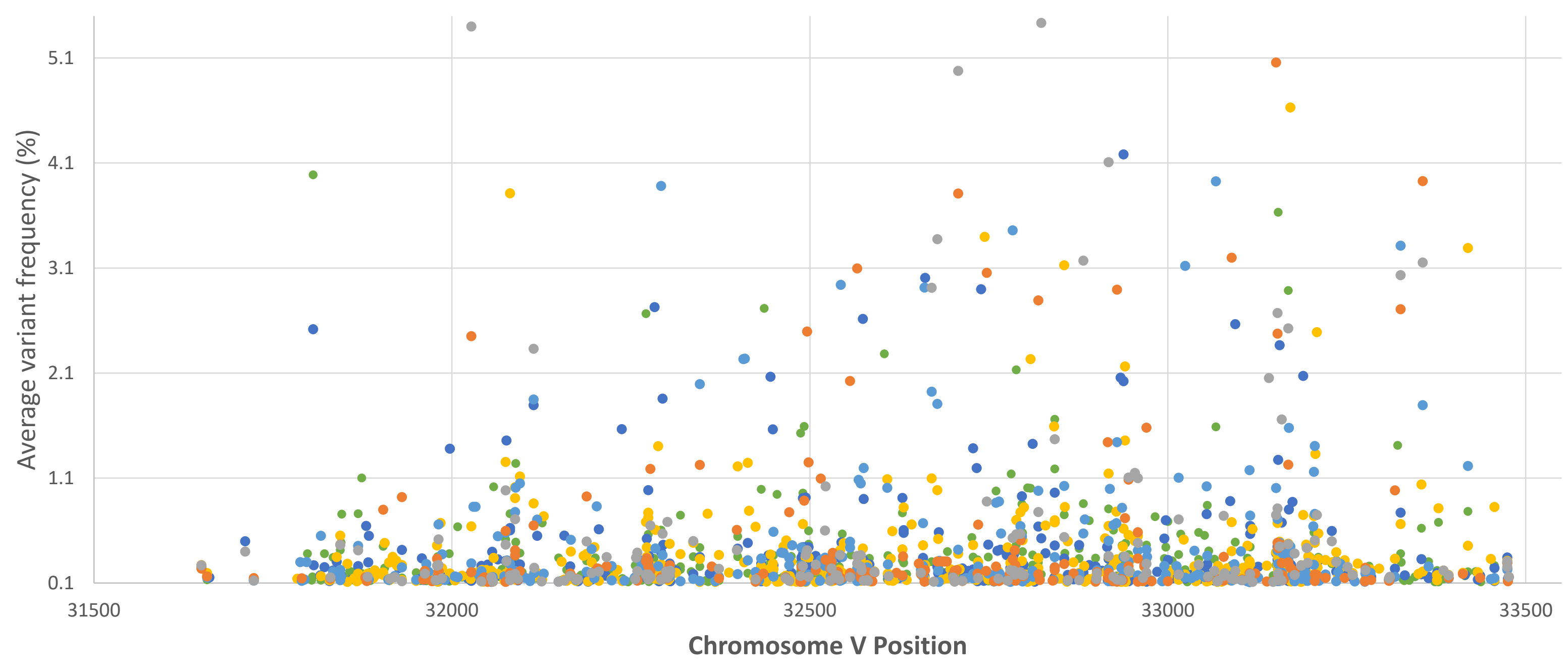

- RNR1

- rnr1D57N

- rnr1Y285F pGAL-RNR1

- rnr1Y285F

- rnr1Y285A pGAL-RNR1

- rnr1Y285A

CAN1 
A

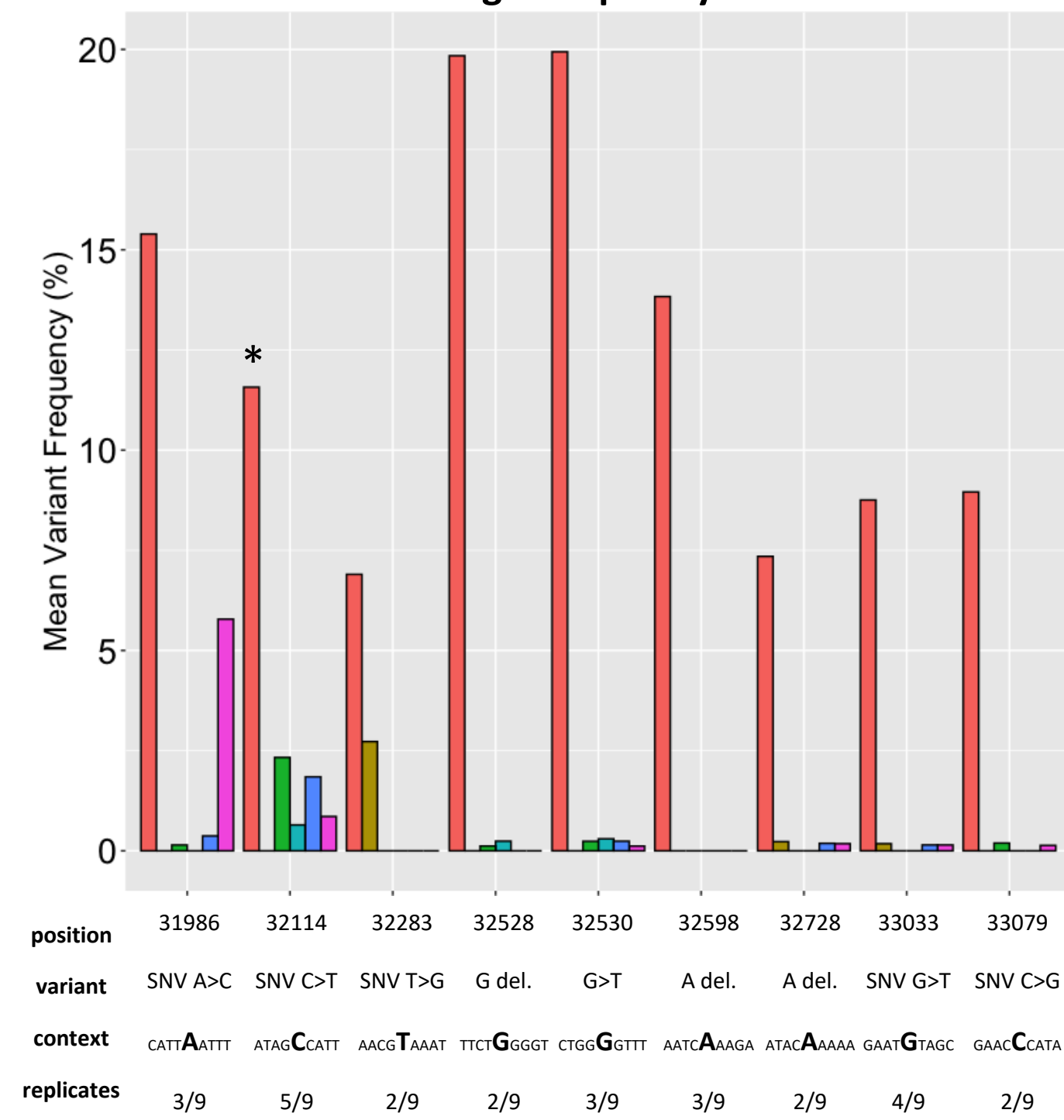

B

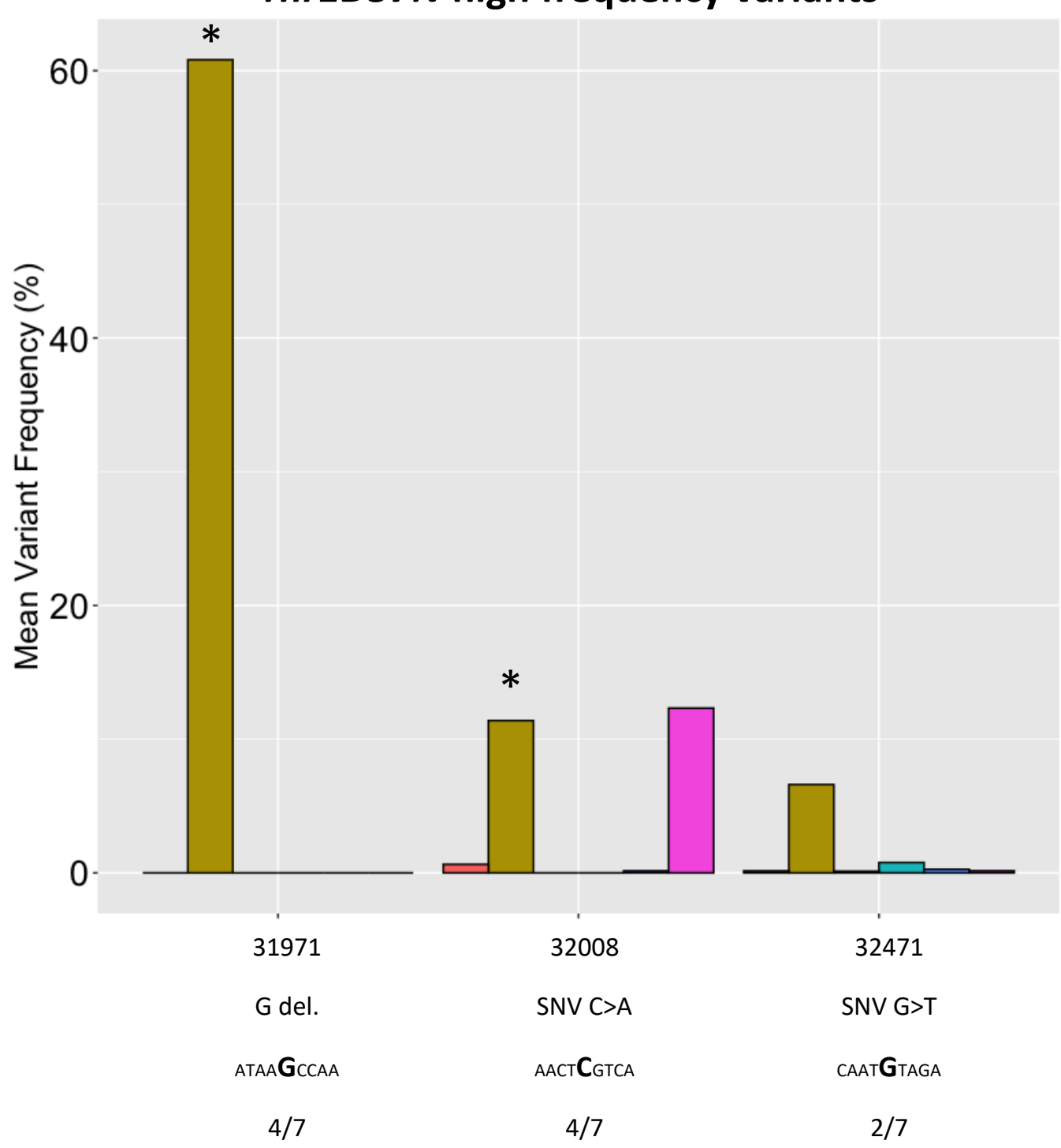

rnr1D57N high frequency variants

Figure 6. 
C rnr1Y285F high frequency variants

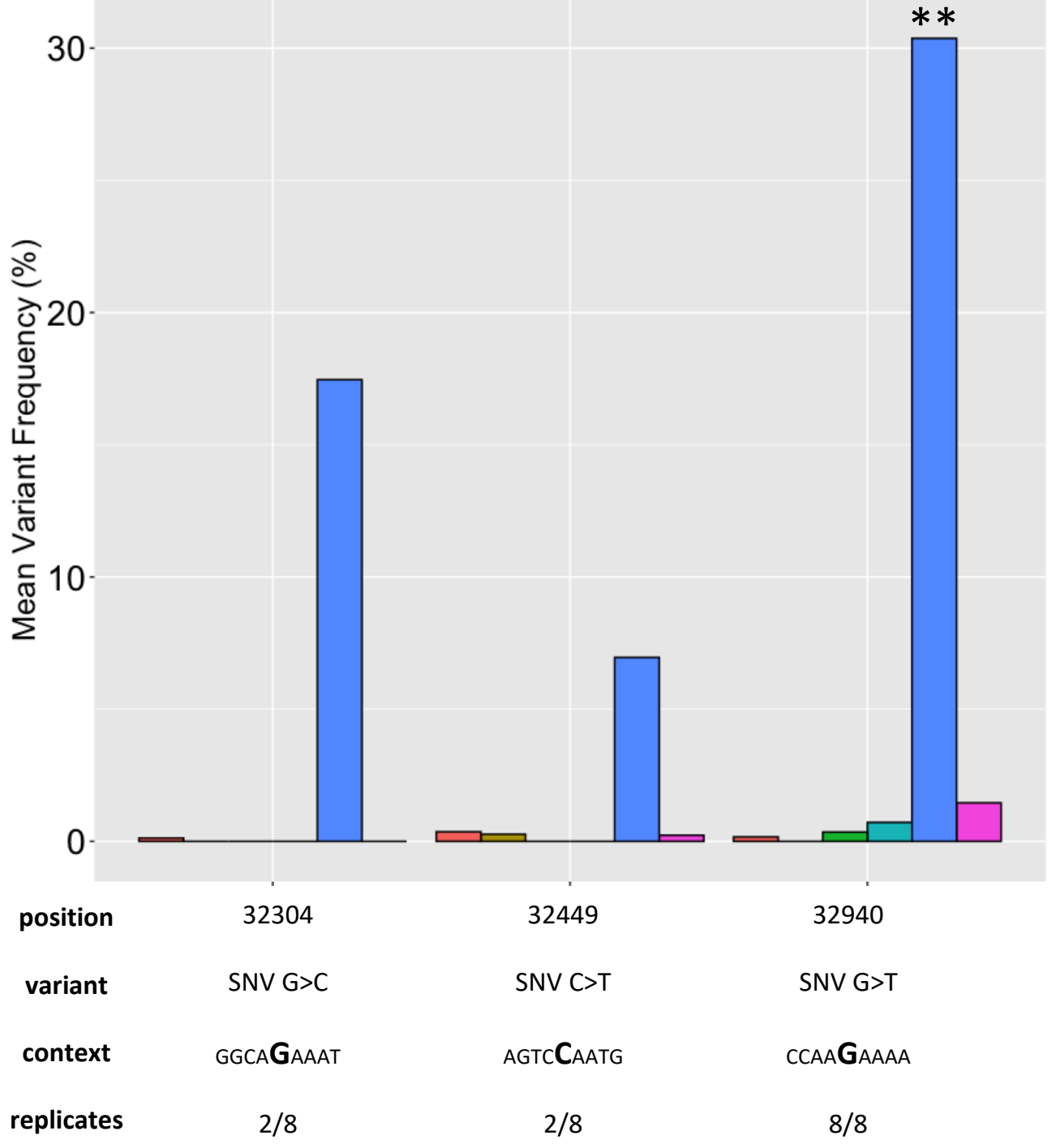

D rnr1Y285F pGAL-RNR1 high frequency variants

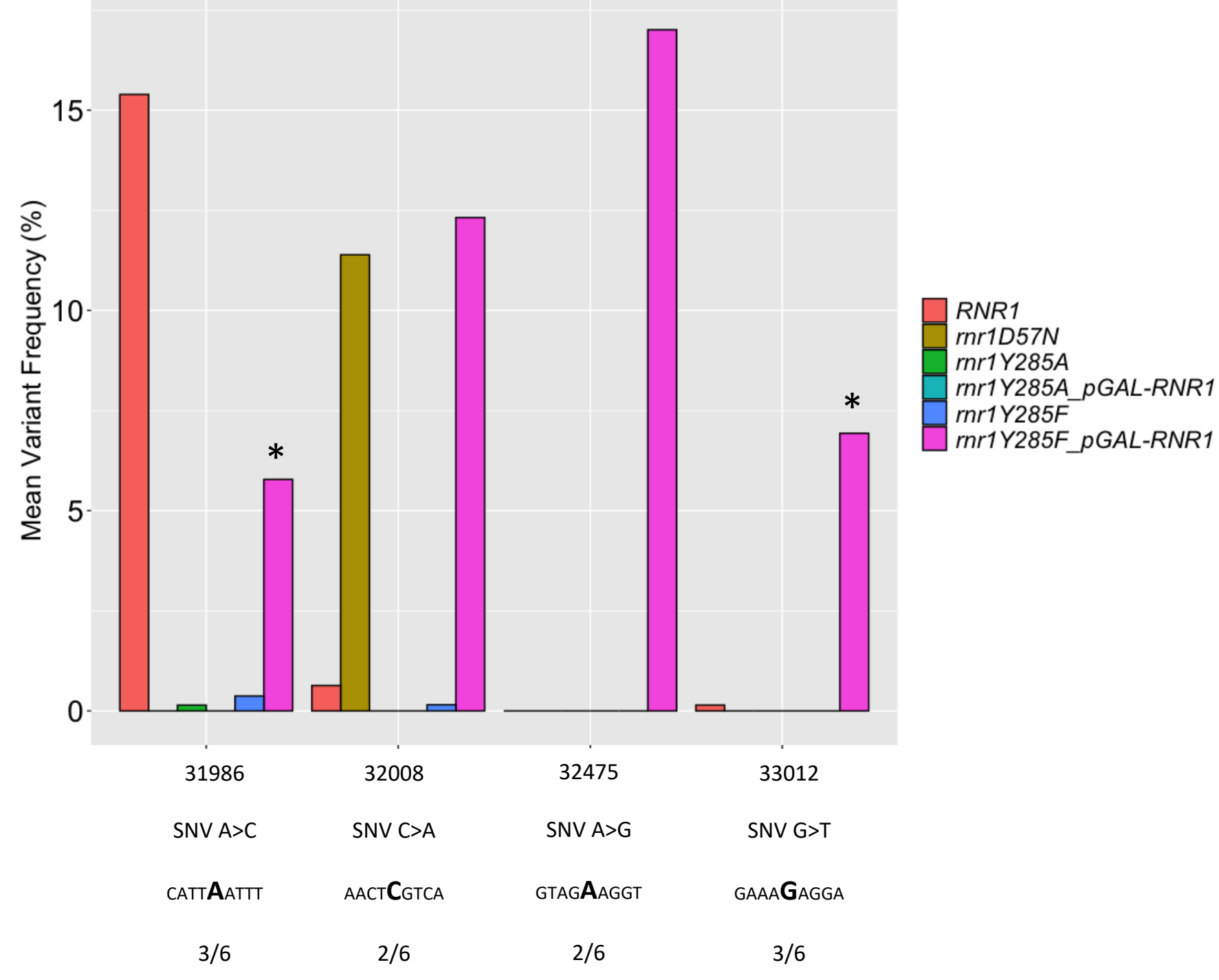

Figure 6 (cont'd). 


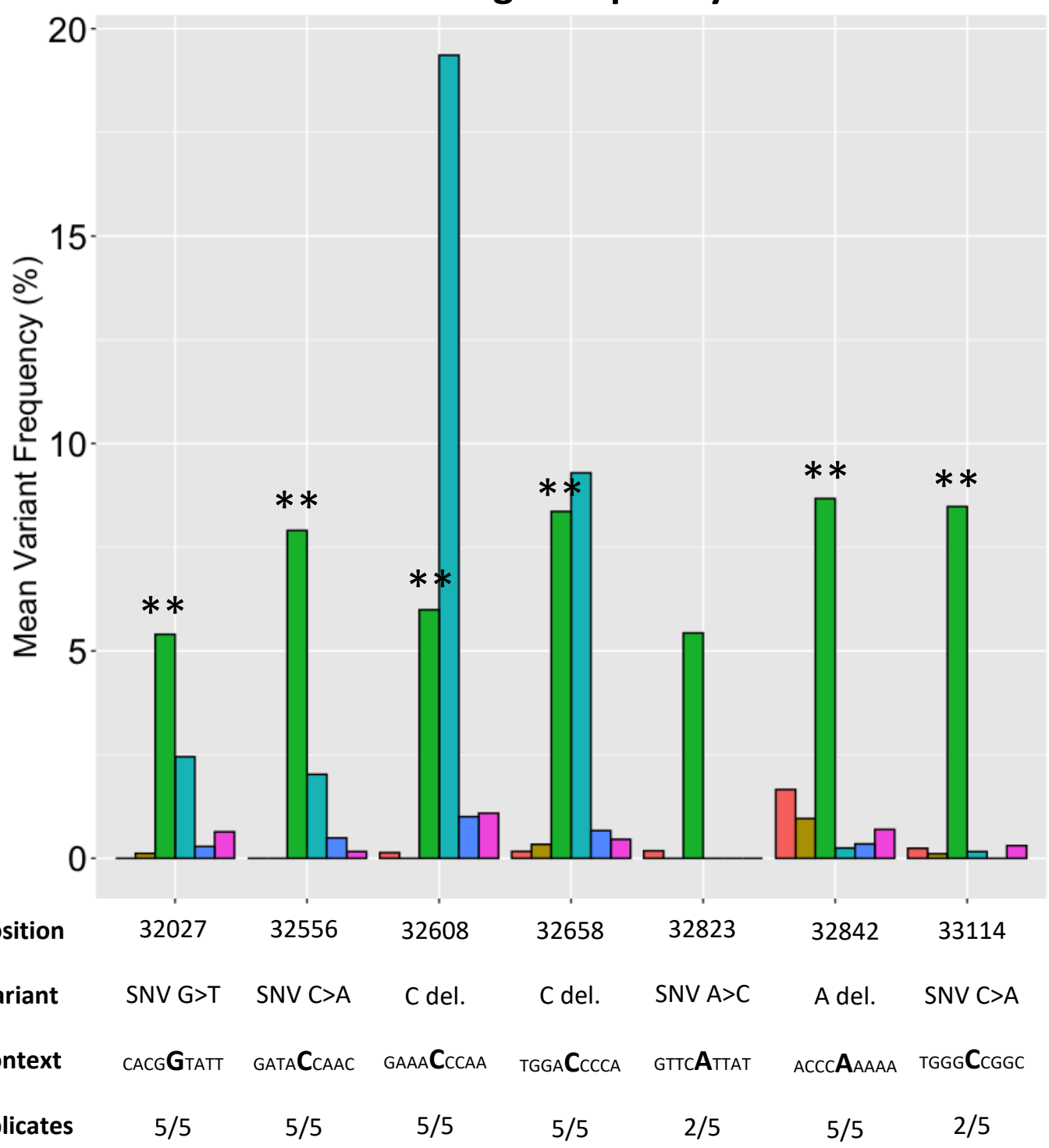

F

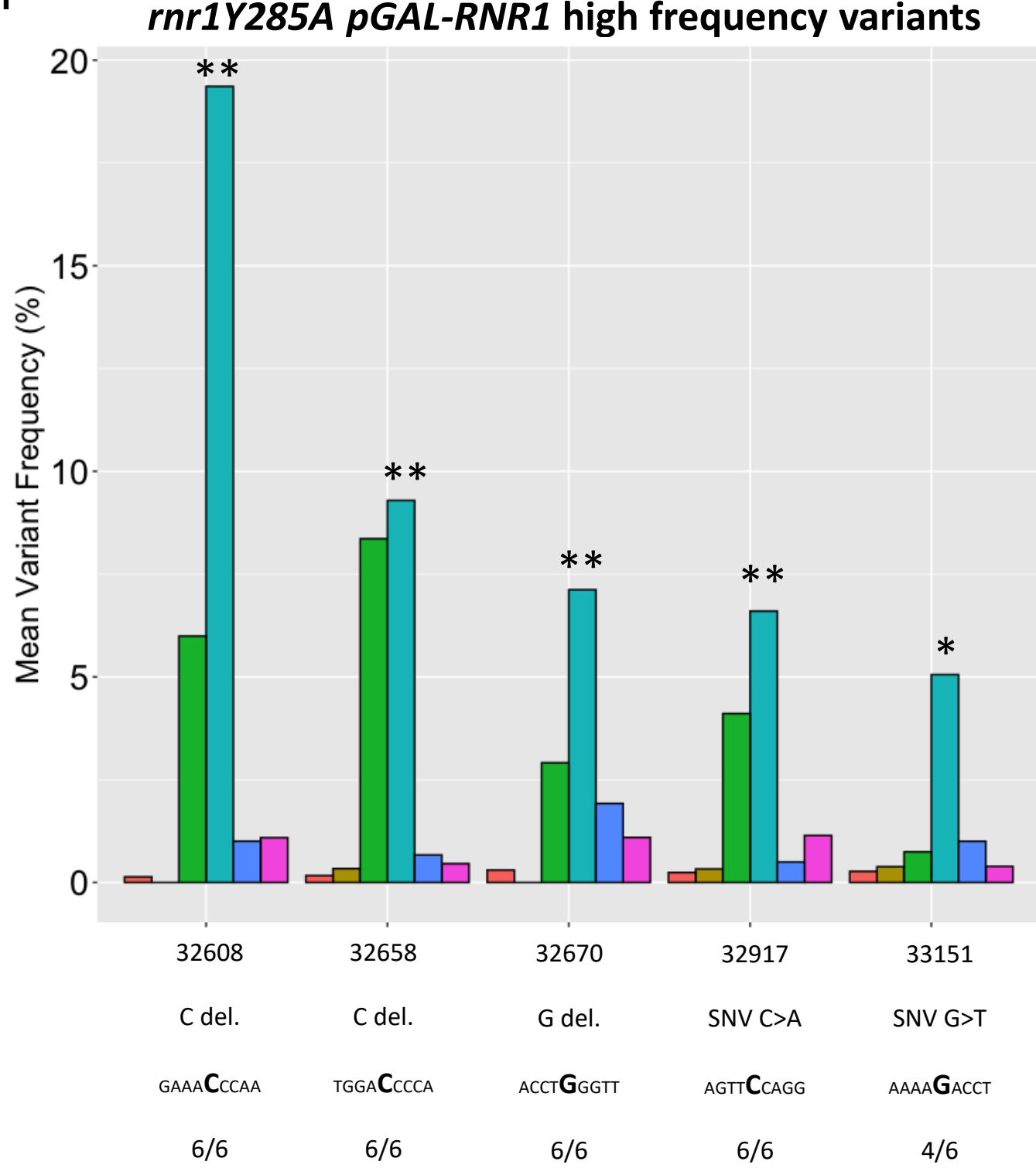

RNR1

rnr1D57N

rnr1Y285A

inr1Y285A pGAL-RNR1

rnr1Y285F

rnr1Y285F_pGAL-RNR1

\section{Figure 6 (cont'd).}




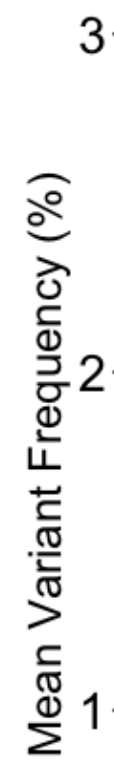

variant

context

0.

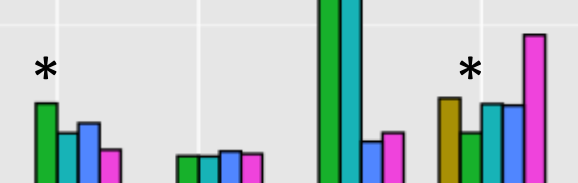

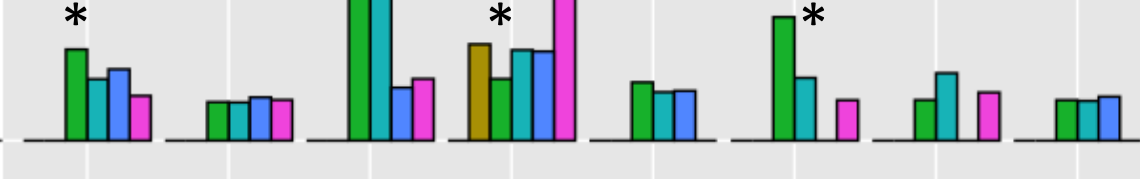

$\begin{array}{lllllllll}31981 & 32057 & 32277 & 32304 & 32538 & 32571 & 32581 & 32713 & 32783\end{array}$

$* *$

$* *$

$\pi^{*}$

$*$

$S N V G>T \quad S N V C>T \quad S N V C>A \quad S N V G>T \quad S N V G>T$ SNV C>A $\quad S N V T>G \quad S N V C>T \quad S N V G>T \quad S N V C>A \quad S N V T>G \quad S N V C>A \quad S N V C>A \quad S N V C>A \quad S N V C>A \quad S N V G>A$

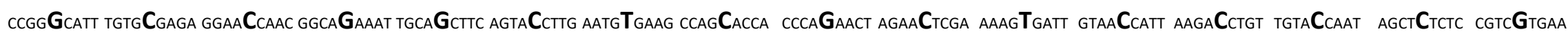

rnr1Y285A replicates

$2 / 6$

$2 / 6$

$4 / 5$

$3 / 5$

$1 / 5$

$1 / 5$

$2 / 5$

$1 / 5$

$5 / 5$

$3 / 5$

$5 / 6$

$2 / 6$

$1 / 6$

Figure 7 (cont'd).
RNR1

rnr1D57N

rnr1Y285A

rnr1Y285A_pGAL-RNR1

rnr1Y285F

rnr1Y285F_pGAL-RNR1 
